\title{
Systematic Literature Review on The Application of Data Mining Methods to Monitor the Operator's Functional State in Human-Machine Systems
}

Marzieh Sadeghian, Assistant Professor, Department of Occupational Health, Faculty of Health, Ahvaz Jundishapur University of Medical Sciences, Ahvaz, Iran.

Soroor Shekarizadeh, Msc, Department of Artificial Intelligence, Faculty of Computer Engineering, Amirkabir University of Technology, Tehran, Iran.

(1) Zahra Mohammadi, ( ${ }^{\star}$ Corresponding author), PhD student, Department of Occupational Health and Safety, Student Research Committee, School of Public Health and Safety, Shahid Beheshti University of Medical Science, Tehran, Iran.

z-mohammadi@sbmu.ac.ir

\section{Abstract}

Background and aims: Continuous monitoring of operator functional state, is one of the most important topics, and the data mining methods are considered as a suitable tool for providing the performance evaluation models. However, there has been no comprehensive study on the use of data mining methods in this field so far, and in so doing, the aim of the present article, was to systematically review the role and importance of the data mining methods to monitor the operator functional state in human-machine systems.

Methods: A total of 86 published articles that evaluated the operator functional state, were reviewed in five databases. All articles were analyzed in four groups related to the operator functional state, three critical safety systems, and three types of data mining techniques. The operator functional state, was also assessed through methods of physical measurement, psychophysiological measurement, task-performance indicators, and subjective judgment.

Results: Most of the data mining models were related to the field of road and air transportation, which are mainly focused on fatigue and the task-performance indicators. The support vector machine and neural network models were the most frequently used data mining methods. The results showed, that most studies were performed on fatigue models, among the functional states where mainly physical measurements were used; however, the psychophysiological measurements were the most frequently applied measuring method for the mental workload models and the task performance indicators.

Conclusion: A comprehensive evaluation of the data mining methods and the parameters used in these models to assess the operator functional state, will identify the research gaps in this area, and results in conducting more extensive studies to improve the human performance.

Conflicts of interest: None

Funding: None

\section{Keywords}

Human-machine system

Data Mining

Operator functional state

Received: 2019/03/15

Accepted : 2020/02/21 


\section{INTRODUCTION}

In human-machine systems, the human operator interacts with the machine and the two sides interact with each other. In fact, the task of humans is determined in relation to the task of the machine, and there is a significant relationship between the motor and sensory force of an individual with the capabilities of the machine. This interaction is in the form of a closedloop system in which, human plays an important role in decision-making. In addition, the complexity of this interaction and the poor system design can cause many faults and accidents $[1,2]$. Therefore, increasing the complexity of the human-machine systems, as well as reducing the physical aspects of job activities, has increased the operator's interaction with the machine, and requires the operators' multi-tasking in the implementation and monitoring of the operations. For this reason, continuous monitoring of the operator's performance is important as one of the requirements of the human-machine systems. The Operator Functional State (OFS), is a multidimensional model of human psychophysiological status that correlates the performance with the task requirements, such as physiological and psychological costs of a work. In general, there are three parts in measuring the operator's functional state, including the mental reporting or judgment, measurement of the task performance indicators, and the physical and physiological measurements. The amazing progress of computer technology has led to the development of data collection and the creation of large databases in various fields, where data mining is a process of extracting information in order to achieve regular and logical patterns. The use of data mining in operator functional state's evaluation models, improves the stability and accuracy of the evaluation results, and is used as an auxiliary process to identify and predict the performance in human-machine systems. However, so far, no comprehensive research has been done on the use of data mining methods in this area. Therefore, the purpose of this study was to systematically review the role and importance of data mining methods to assess the operator functional state in human-machine systems. In this study, a framework was presented, that in addition to providing a comprehensive view of the application of data mining models and methods to evaluate the operator's function, also identifies the main application areas of these methods and measurement parameters.

\section{METHODOLOGY}

\section{Question design in PICO format}

In this study, are included, population $(\mathrm{P})$ : the operator of human-machine systems, intervention (I): the use of data mining, and outcome, $(\mathrm{O})$ : the operator functional state.

\section{Inclusion and exclusion criteria}

In this study, 3 inclusion criteria were considered: (1) published articles or studies presented at the conference that used at least one data mining method to monitor the performance of people in the humanmachine systems, (2) articles which have described the data mining methods in detail, (3) data mining methods were quite recognizable in the context of the article. In addition, the master's or doctoral dissertations, textbooks, and unpublished articles were determined as the exclusion criteria.

\section{Search strategy}

The researches were conducted in five categorize to cover the most scientific journals in the English language. In addition, many researches were performed in terms of the words related to the human functional state, and words related to data mining methods. The time frame was considered between 1999 and 2018

\section{Select articles}

Initially, 587 articles between the years of 1999 to 2018 were identified. Finally, 86 articles were selected according to the systematic framework presented by Hachicha and Ghorbel. A detailed review of each article for one of the following human-machine systems, including transportation, process and power plant systems, one for the following four states of fatigue, distraction, mental workload and task performance indicators, as well as one for the following three classification, prediction, and regression models were done.

\section{Data extraction}

The operator functional state was evaluated in five groups of: distraction, fatigue, mental workload, task performance indicators (speed, accuracy, reaction time, etc.) and hybrid parameters of the mentioned items. The operator functional state's measurement was also divided into five categories: physical measurements, psychophysiological measurements, methods of measuring the task performance indicators, reporting or subjective judgment methods, and a combination of the mentioned measurements. The status of the operator in human-machine systems is examined with different data mining models, which usually includes the classification, prediction and regression models. To review the articles, first, the three main data mining models including regression, classification, and prediction were identified and then, the data mining methods were determined.

\section{Assess study quality}

The quality of the articles in this study, was evaluated based on the STROBE tool. 


\section{RESULT}

Review of the articles, based on the proposed study framework

The results of the article's evaluation showed that, the major data mining models proposed were associated with the transportation systems (58 road transport articles: $67.44 \%$ and 20 air transport articles: $23.25 \%$ ), then, fewer studies related to process and power plant systems were observed (8 articles: $9.3 \%$ ). The most application of data mining methods was observed in road and air transportation systems, the main focus of which was on fatigue ( 36 articles) and the task performance indicators ( 15 articles). Less studies have been done regarding the process and power plant industries; there were 3 articles concerning each of the fields of mental workload and fatigue, and the rest were related to task performance indicators ( 1 article) and the combined cases ( 1 article).
Figure (1), shows the application of measurement methods in the five groups of operator functional states. Based on the results, the models related to the fatigue parameter, had a higher contribution than other areas of the performance status. In fatigue models, the order of using the measurement methods includes: the physical measurements (22 articles: 25.58\%), psychophysiological measurements (12 articles: 13.95 $\%)$, measurement of the task performance indicators (3 Article: $3.49 \%$ ) and combined measurement methods (2 articles: $2.32 \%$ ). The application of measurements for the distraction parameter is also in the form of physical measurement methods including 3 articles (3.49\%) and psychophysiological measurements, measurement of the task performance indicators and combined measurement methods including 2 articles for each $(2.32 \%)$. Psychophysiological measurement methods were most used to construct models for

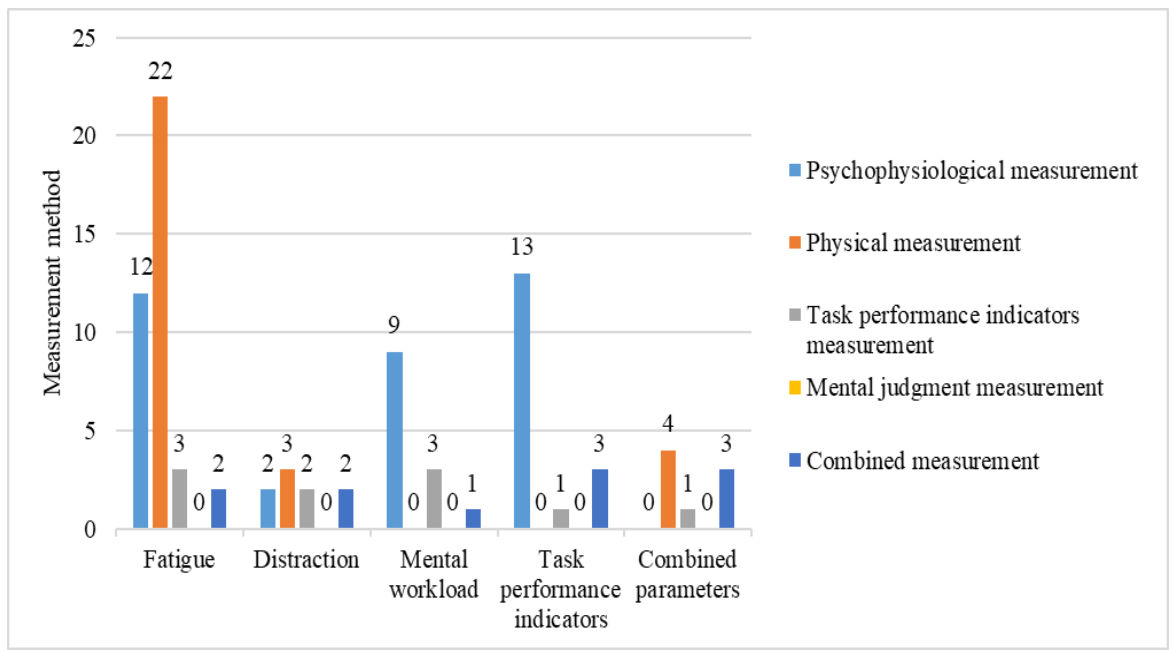

Figure 1. Distribution of the articles, based on the operator functional state measurement methods

Table 1. Distribution of the articles, based on the operator functional state and data mining models

\begin{tabular}{lcc}
\hline Operator functional state & Data mining model & Number of articles \\
\hline & Classification & 24 \\
Fatigue & Prediction & 11 \\
& Regression & 2 \\
& Combined models & 2 \\
Distraction & Classification & 3 \\
& Prediction & 5 \\
Mental workload & Regression & 1 \\
& Classification & 9 \\
Performance indicators & Prediction & 4 \\
& Classification & 5 \\
Combined parameters & Prediction & 12 \\
& Classification & 2 \\
Total & Prediction & 5 \\
\hline
\end{tabular}




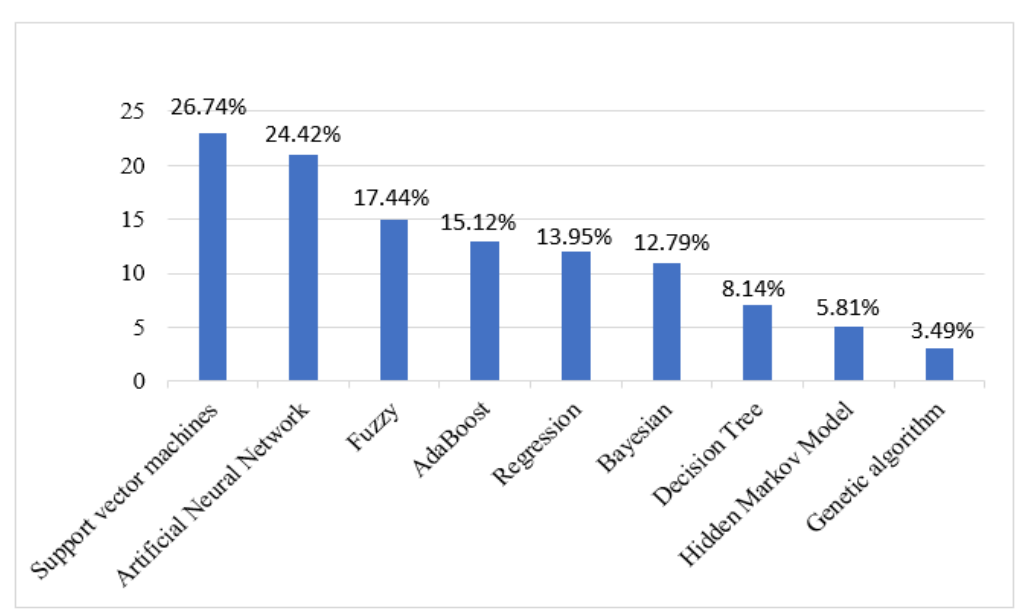

Figure 2. Distribution of the articles, based on the application of data mining methods

mental workload and task performance indicators (9 and 13 articles: $10.46 \%$ and $15.12 \%$, respectively), but in the combined parameters of the performance status, the physical measurement methods were used the most (4 articles: $4.65 \%$ ).

Table (1) shows the distribution of articles based on their functional state and models presented. The classification models were identified as the most widely used data mining models (43 articles), and the models of prediction (37 articles), regression ( 4 articles) and also a combination of data mining models ( 2 articles) were placed in the next ranks.

The main data mining algorithms used to evaluate the operator functional state, are based on Figure (2). The support vector machine (23 articles: $26.74 \%$ ) and neural network (21 articles: $24.42 \%$ ) methods showed the most application in constructing the models for evaluating the operator functional state. Also, the fuzzy model (15 articles: 17.44\%), AdaBoost (13 articles: $15.12 \%)$, regression (12 articles: 13.95\%), Bayesian (11 articles: $12.79 \%)$, decision tree (7 Article: 8.14\%), hidden Markov model (5 articles: $5.81 \%$ ) and genetic algorithm (3 articles: $3.49 \%$ ), were in the next categories.

Distribution of the articles by the year of publication

Articles on the application of data mining methods for operator functional state, from 1999 to 2009, had an increasing trend, such that the largest number of articles in this field was published in 2009. After 2009, the publish rate decreased compared to this year.

\section{Distribution of the articles by the country name}

Among the reviewed articles, most of the studies were related to the United States and China (22 and 21 articles, respectively). Other articles have been done in different countries such as Australia, England, Korea, etc.

\section{DISCUSSION}

According to the results, among the three humanmachine systems examined in this study, the researchers paid the most attention to transportation systems (90.7\% of articles). The most researches conducted to road and air transportation systems, focused on fatigue and performance indicators, respectively. While fewer of the models presented, deal with the distraction and mental workload. Therefore, considering their high impact on the occurrence of accidents, further researches on the study of the distraction and mental workload parameters is suggested. On the other hand, despite the relatively few studies conducted to the power plant and process industries, as well as the importance of the human performance role in these systems, it is important to conduct more extensive studies and further develop the proposed models. In most models, physical and psychophysiological measurement methods have been used that, more applications of, can be due to higher sensitivity and thus providing more accurate models. In the present study, the results of examining the types of data mining techniques used to determine the operator functional state, showed that the classification models and then predictive models are the most widely used models in these studies. In addition, considering that the classification and prediction models provide an effective and useful prediction of human functional state (as a complex system) by examining the operator's behavior, the high application of these models would not be far from expectation. On the other hand, the support vector machine method and then the neural network, were the most used among other models. The support vector machine algorithm provides more accurate classification results than other common methods and is used for many classification problems. In addition, due to the multiplicity of parameters in the discussion of the operator functional state, the 
complexity of the relationships between them, and the existence of unknown interfering parameters, the selection of an appropriate method to properly evaluate the relationships between this categories, should be carefully considered. Therefore, the use of nonlinear relationships such as neural network models is one of the solutions recommended by various researchers in this field; and in recent years has attracted the attention of the scientific community. In fact, the neural network has a high ability to detect complex nonlinear relationships between dependent and independent parameters [122, 124], and is able to identify the possible interferences between the predicted parameters.

The review of articles also showed that the United States and China have paid special attention to the use of data mining in the studies of human performance, but in Iran, the number of articles was very low. Considering the statistics of the transportation accidents, power plant and process systems and the role of human performance in the occurrence of these accidents [125-127], it is necessary to examine the role of various factors on the human performance in Iran, using the data mining techniques and models to purposefully control the accidents.

\section{CONCLUSIONS}

Due to the complexity of job tasks and concerns about the consequences of possible functional defects, accurate estimation of the user's performance and determination of the conditions affecting it, leads to the integration of the human-machine system interaction. Various models have been proposed to assess the human functional state that, the general approach of these models is to pay attention to the complexity of job tasks and reduce concerns about the consequences of operator's functional defects in critical safety situations. The important point in using the models, is their accuracy of prediction, thus, higher accuracy models provide a more accurate assessment of the operator's performance and the possibility of achieving the optimal condition. In addition, these models are more accepted by the system designers and users. In recent years, data mining methods have been widely used to model the operator's performance in human-machine systems, that the support vector machine method and then the neural network had the highest application among other models. Therefore, in future studies, it is necessary to examine the efficiency and results of each of the different data mining methods in this field.

In general, a comprehensive evaluation of datamining-based models, in order to determine the operator functional state in human-machine systems and identify performance-related factors and measurement methods in various articles, will lead to the identification of a research gap in this area, and the conduction of more researches to assess and improve the state of human performance.

\section{CONFLICT OF INTEREST}

The authors declare that there are no conflicts of interest regarding the publication of this manuscript.

How to cite this article:

Marzieh Sadeghian, Soroor Shekarizadeh, Zahra Mohammadi. Systematic Literature Review on The Application of Data Mining Methods to Monitor the Operator's Functional State in Human-Machine Systems. Iran Occupational Health. 2021 (01 Sep);18:17.

*This work is published under CC BY-NC 4.0 licence 


\section{مرور سيستماتيك كاربرد روش هاى داده كاوى جهت هايش وضعيت عملكرد إيراتور در سيستم هاى انسان - ماشين}

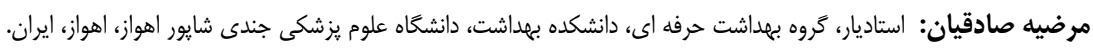

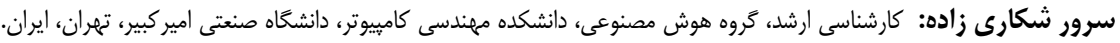

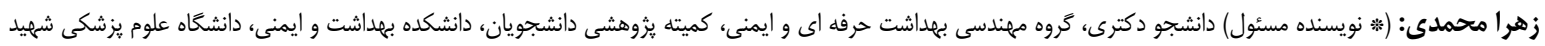

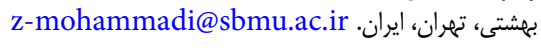

جكيده

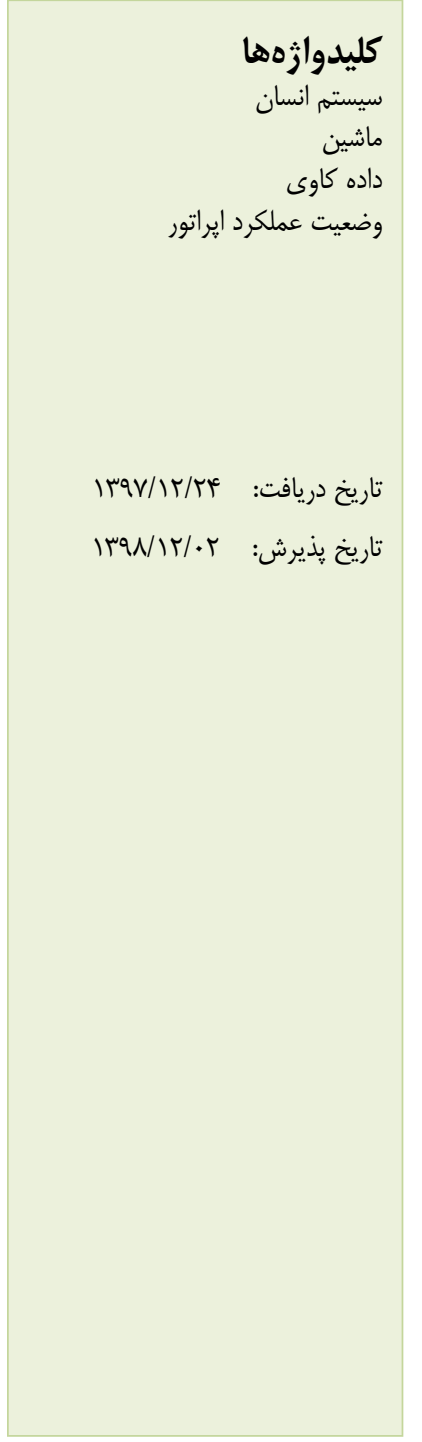

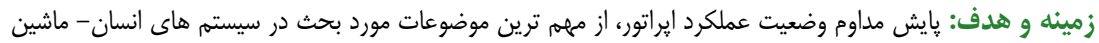

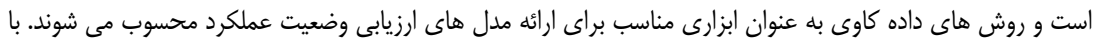

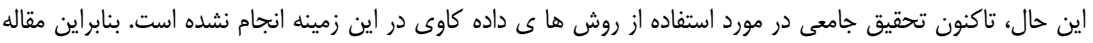

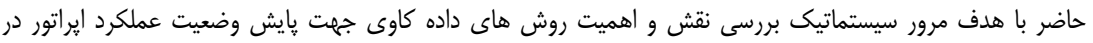
سيستم هاى انسان - ماشين انجام شده است.

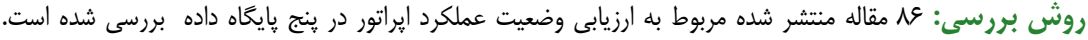

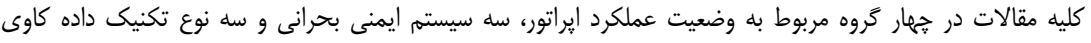

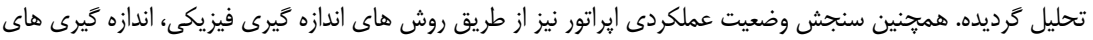

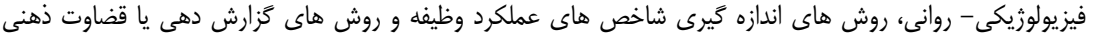
مورد بررسى قرار كرفته است.

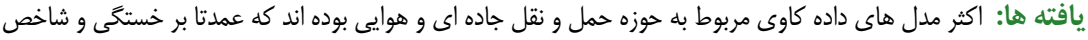

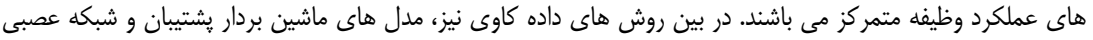

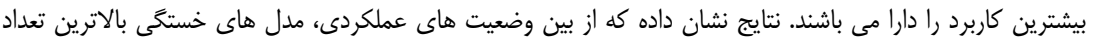

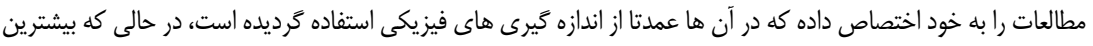

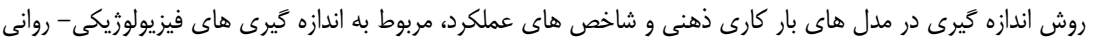
مى باشد.

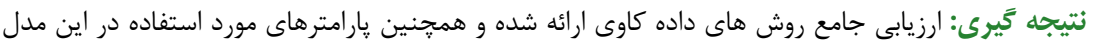

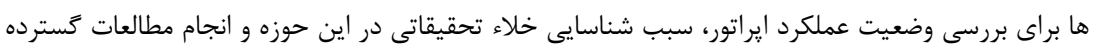

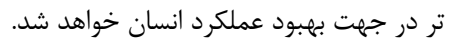
تنعارض منافع: كزارش نشده است. منبع حما يت كنتنده: ندارد.

شيوه استناد به اين مقاله: Marzieh Sadeghian, Soroor Shekarizadeh, Zahra Mohammadi. Systematic Literature Review on The Application of Data Mining Methods to Monitor the Operator's Functional State in Human-Machine Systems. Iran Occupational Health. 2021 (01 Sep);18:17. 
قلب، فشار خون، سرعت تنفس، حركات يلك، فعاليت

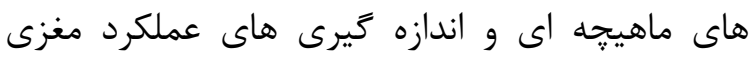

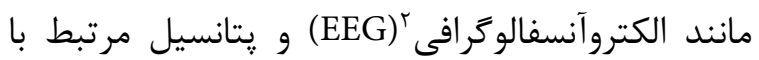

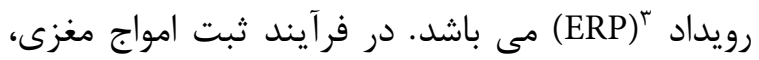

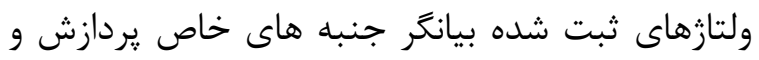

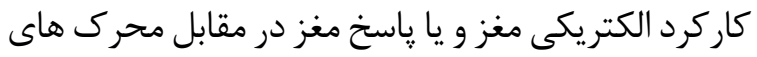

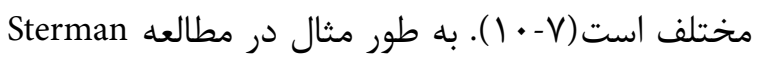

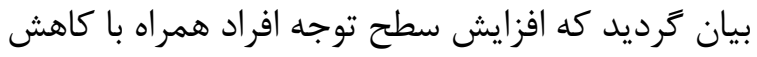

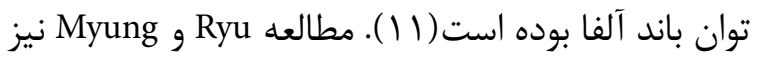

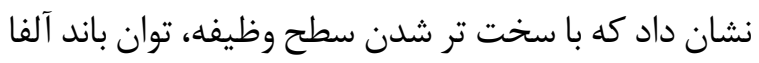

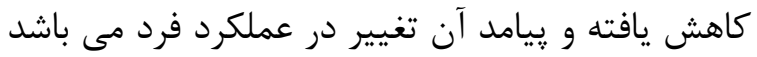

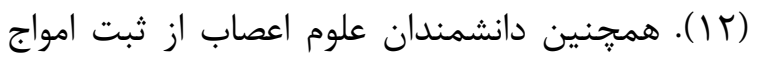

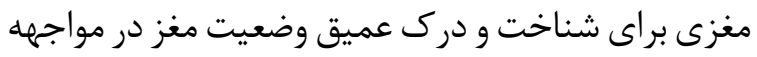

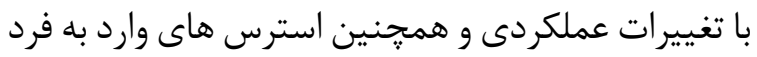

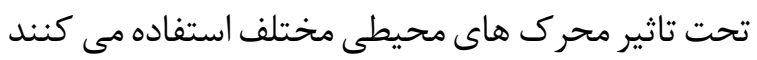

(1 $(1,1 \cdot-v)$

ارزيابى وضعيت عملكرد إيراتور توسط يارامترهايى مانند

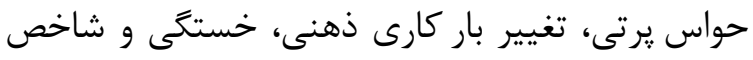

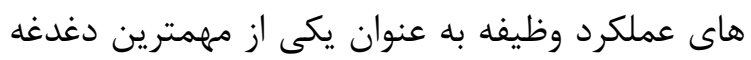

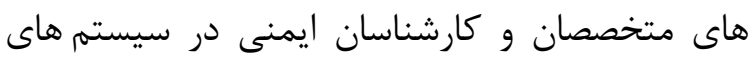

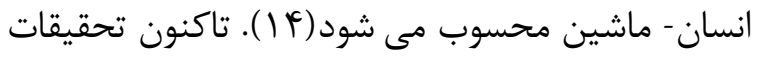

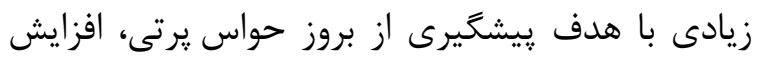

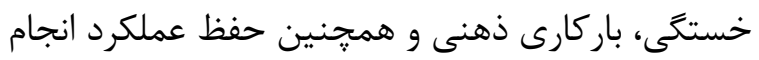

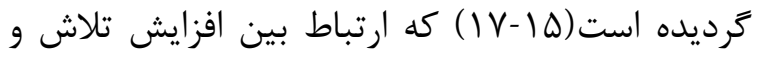

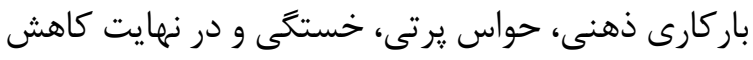

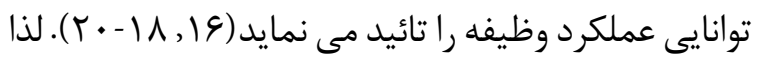

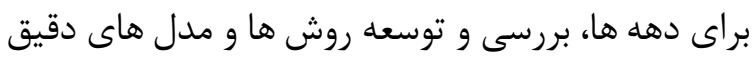

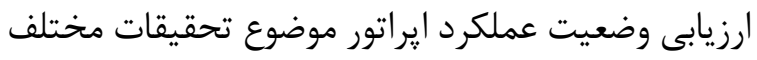

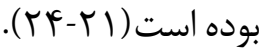

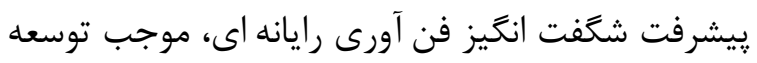

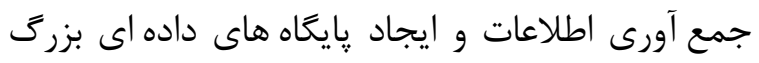

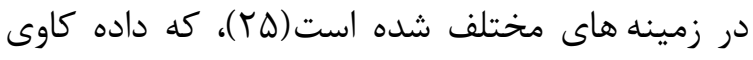

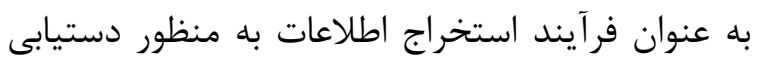

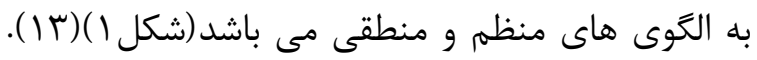

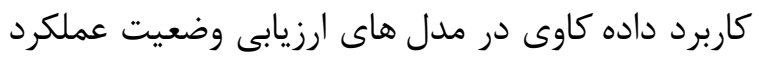

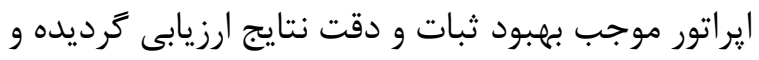

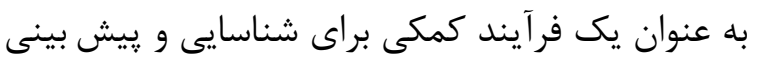

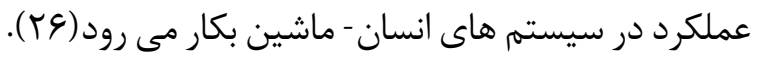

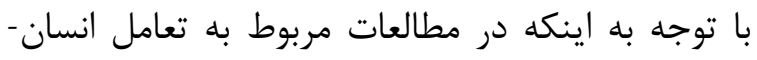

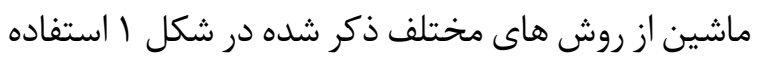

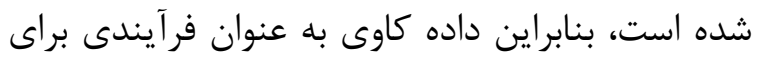

2 Electroencephalography

3 Event-related potential

در سيستم هاى انسان - ماشين، إيراتور انسانى با ماشين

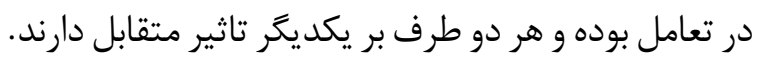

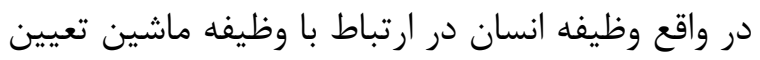

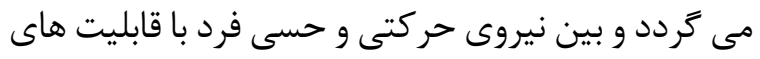

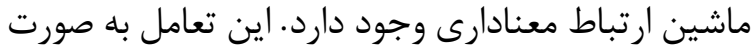

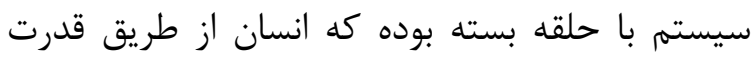

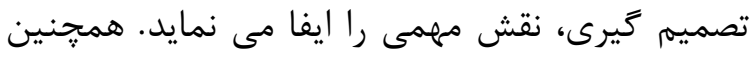

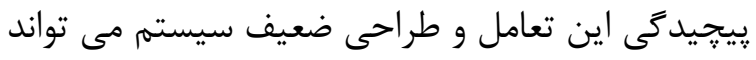

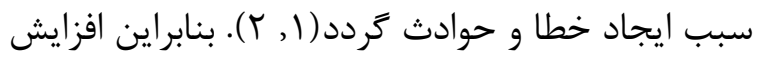

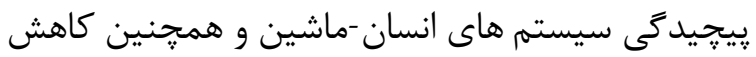

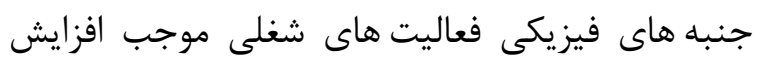

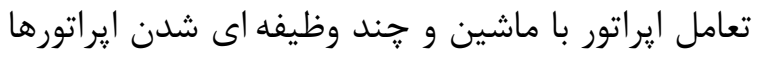

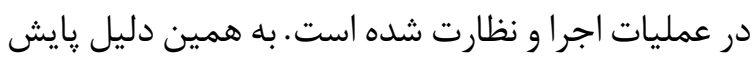

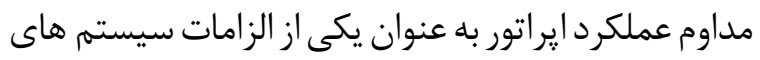

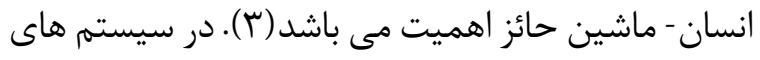

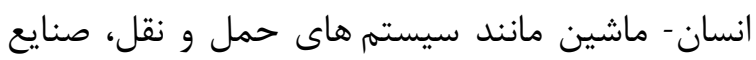

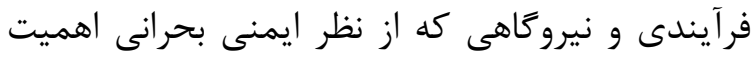

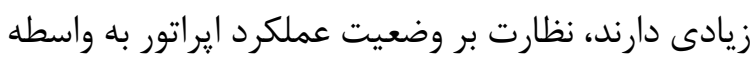

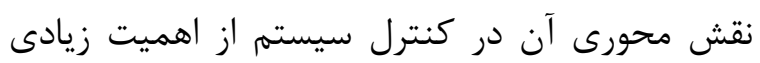

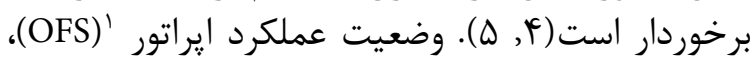

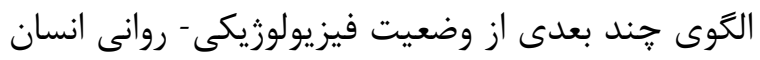

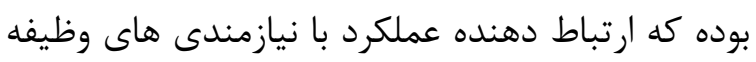

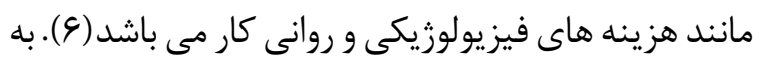

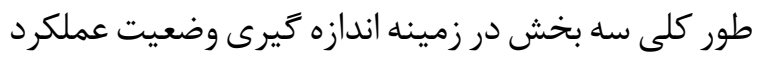

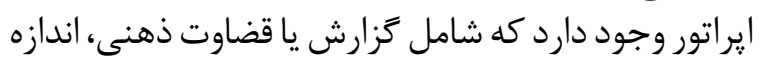

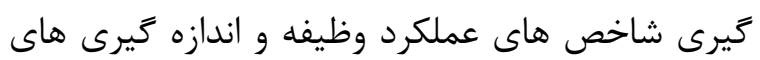

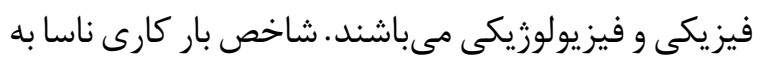

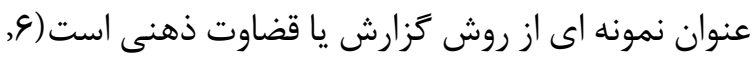

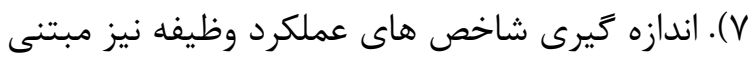

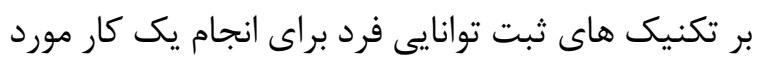

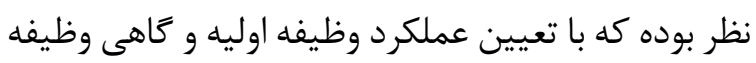

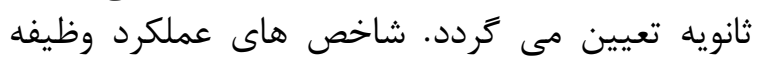

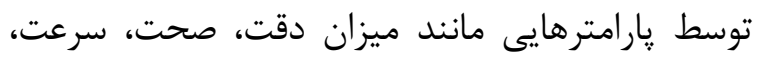

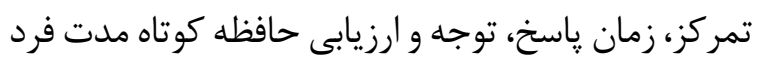

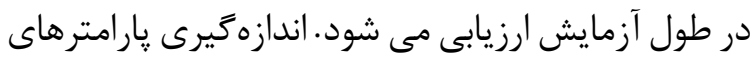

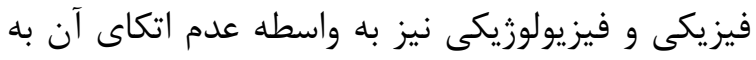

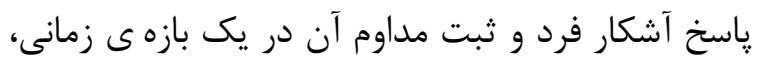

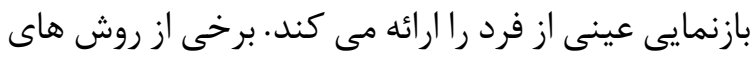

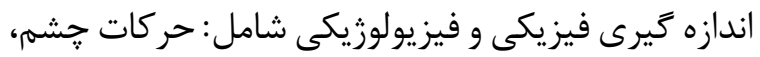

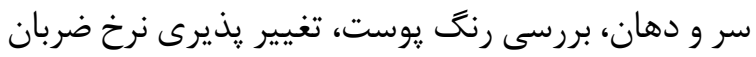

1 Operator functional state

Iran Occupational Health. 2021 (01 Sep);18: 17. 


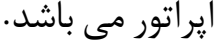 \\ معيارهاى ورود وخروج}

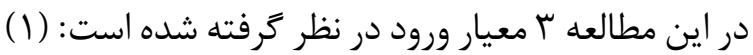

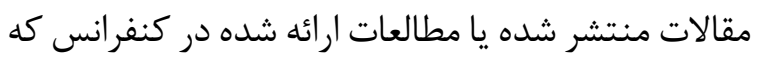

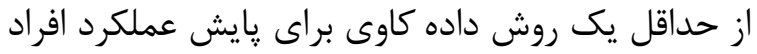

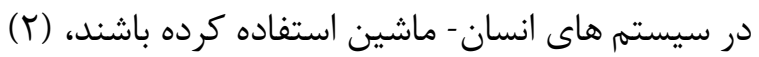

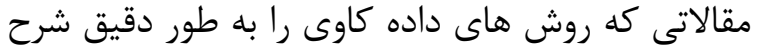

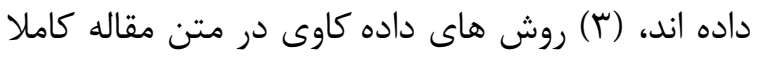

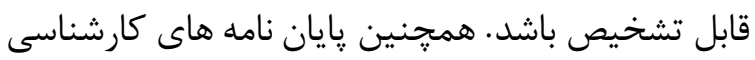

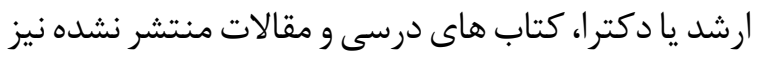

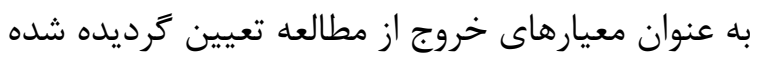

$$
\text { استراتثى جستجوى متون }
$$

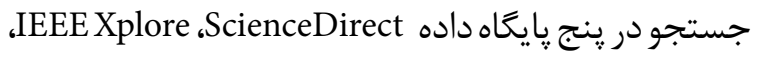
عال Siley SpringerLink ، Scopus علمى موجود در زبان انخليسى را يوشش مى دهى دهند، انجام

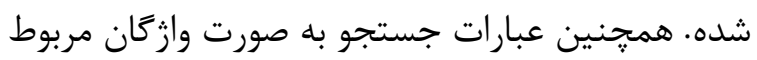
به وضعيت عملكرد انسان شامل: Mental، هistraction، Operator ،Drowsiness ،Tiredness ،Fatigue Workload state ،Mental performance “functional و وازكان مرتبط با روش هاى داده كاوى شامل: Data Hidden ،Neural network ،Bayesian network ، mining Support vector ،Regression ،Decision tree ،Markov K-nearest،Genetic Algorithm ،Adaboost ،machine Prediction, Classification ،Clustreing ،neighbor بوده است. جهت تعيين كليدوازه هاى مربوط به تكنيك

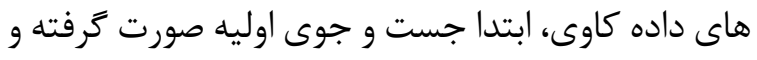

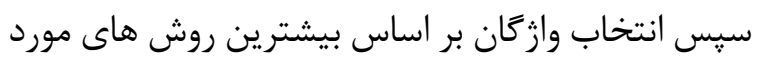

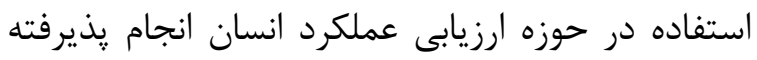

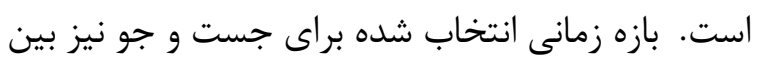

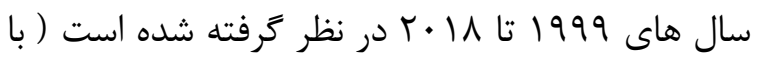

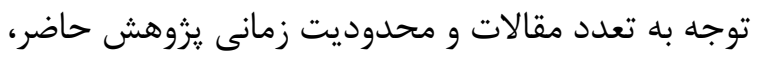
نويسند

$$
\text { برسيى مقالات }
$$

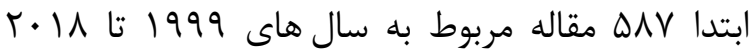

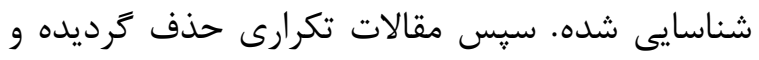

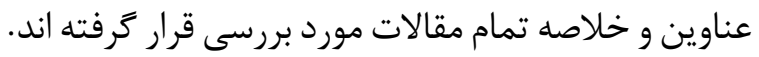

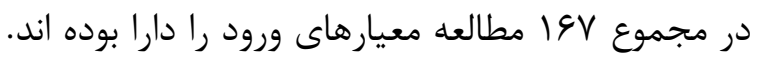

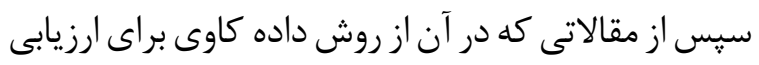

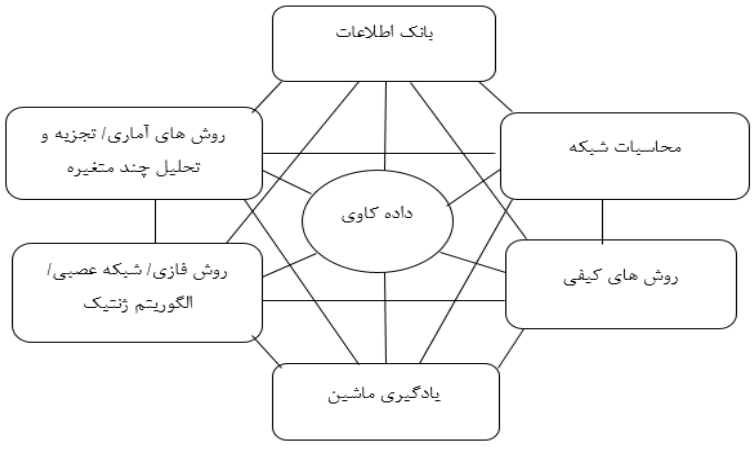

شكلا. داده كاوى در زمينه هاى مختلف(با)

استخراج اطلاعات و الكوهاى مفيد از رايغاه هاى داده ،

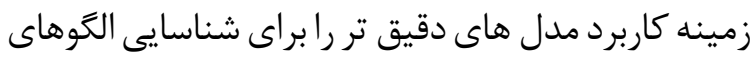

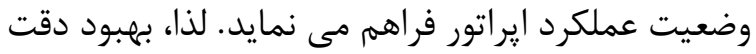

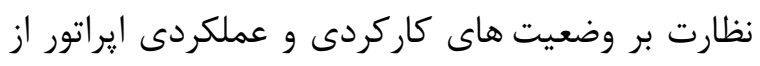

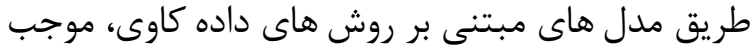

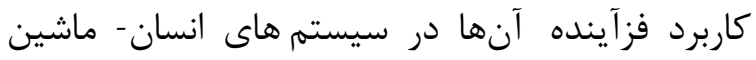

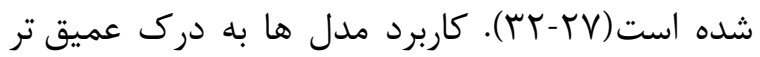

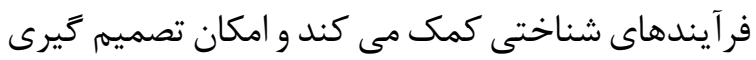

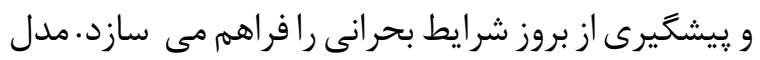

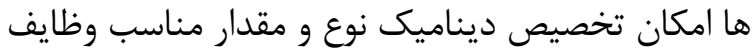

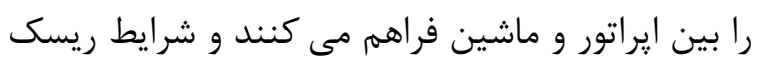

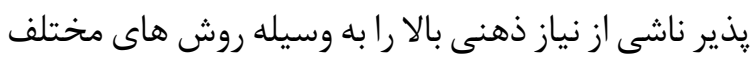

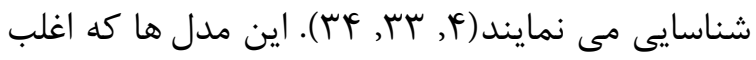

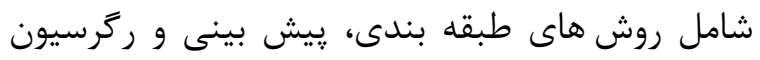

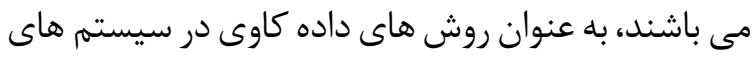

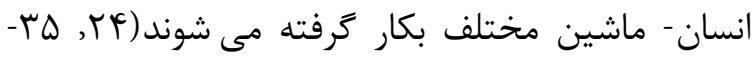
TV

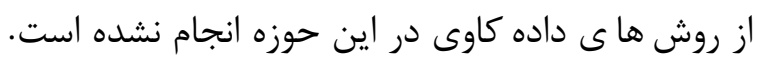

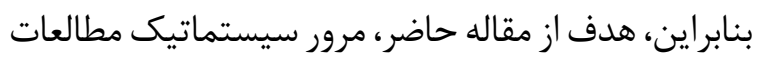

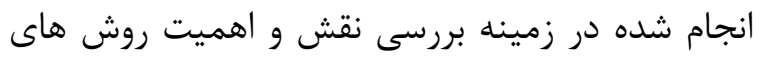

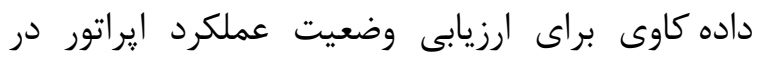

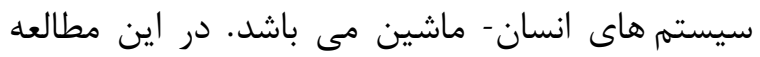

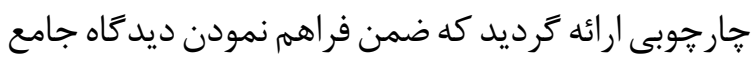

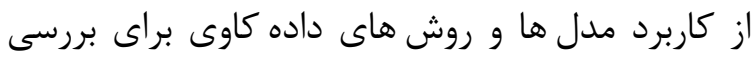

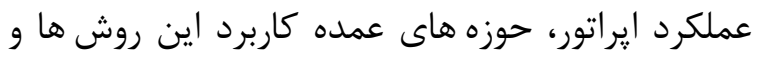

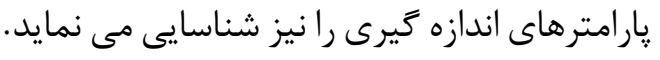

$$
\text { طراحى سوال درقى رالب دICO }
$$

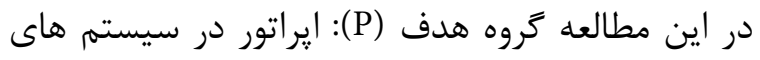

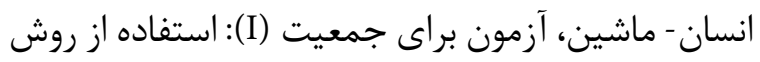
هاى داده كاوى و خروجى (O): شامل وضعيت آزيت عملكرد 


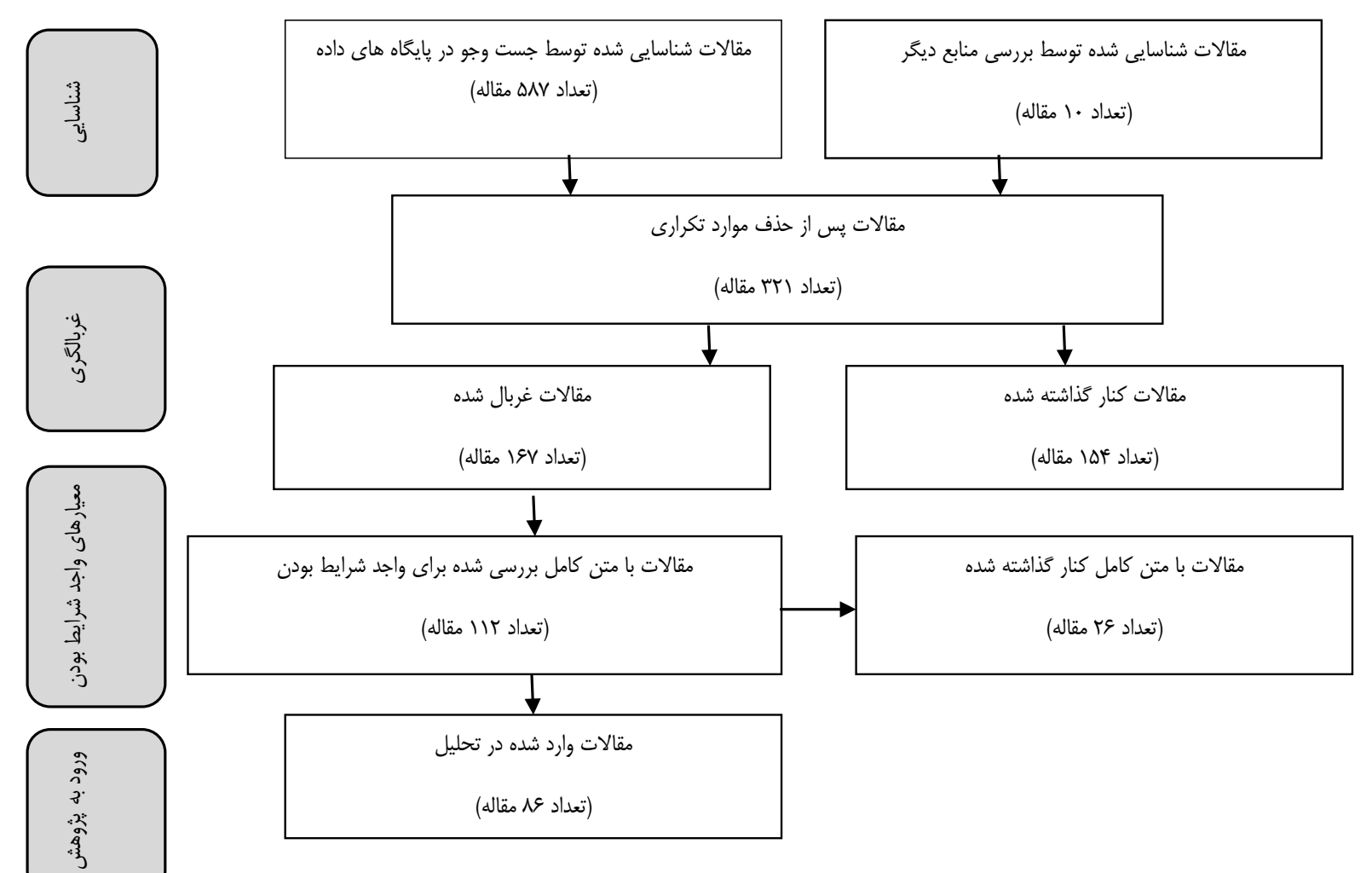

شكل r. خلاصه مراحل انجام مطالعه مرورى

و رگرسيون انجام يذيرفته است.

استخراج داده ها

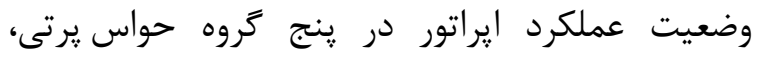

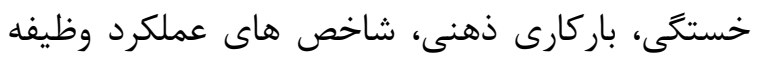

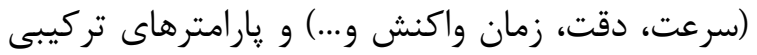

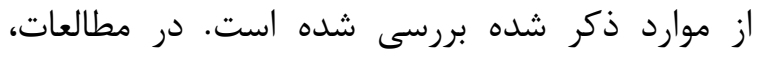
حواس يرتى اغلب به عنوان ناتوانى در تمركز و عامل

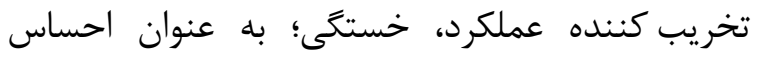
ذهنى خستخى و كاهش دهنده عملكرد ذهنى يا فيزيكى، باركارى ذهنى؛ نسبتى از ظرفيت روانى فرد براى انى انجام

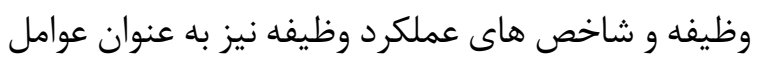

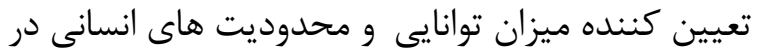

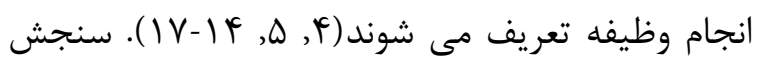

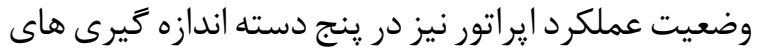

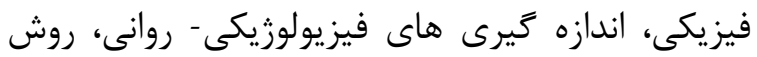

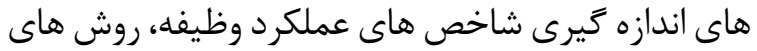

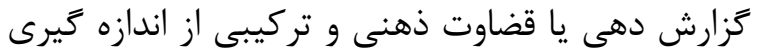

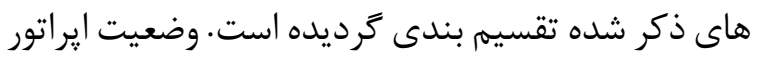

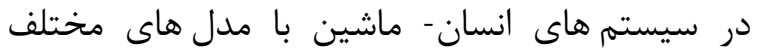

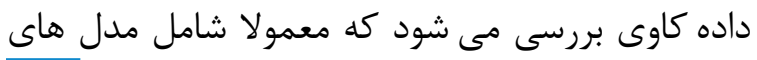

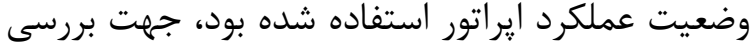

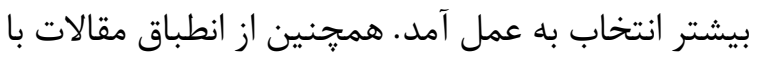

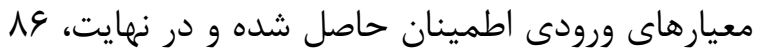
مقاله با توجه به جار جوب وردي سيستماتيك ارائه شده توسط Hachicha برنامه جارجوب سيستماتيك كه در اين مطالعه اتخاذ

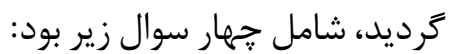

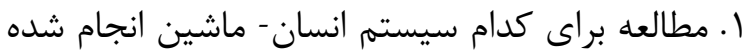
است؟ r. كدام وضعيت عملكردى ايراتور ارزيابى شده و از جه إنه

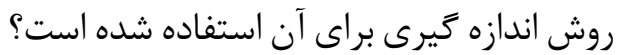
ץ. جه تكنيك داده كاوى استفاده شده است؟

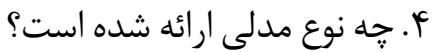
فرآيند ارزيابى مقالات به طور مستقل و دقيق دقيق به وسيله

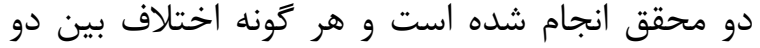

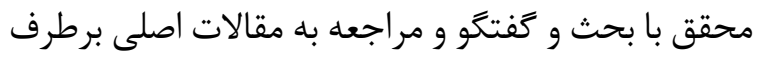

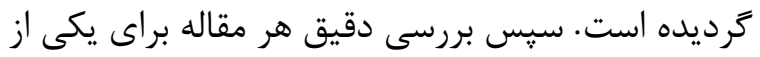

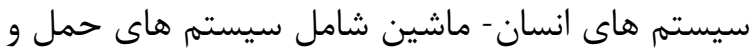

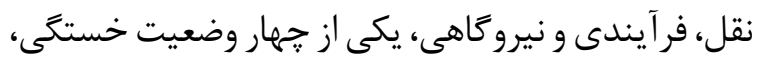

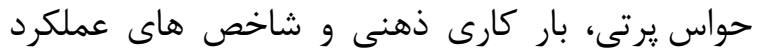

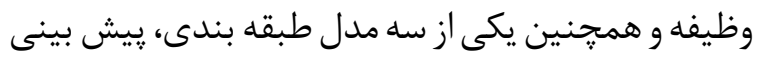
Iran Occupational Health. 2021 (01 Sep);18: 17. 


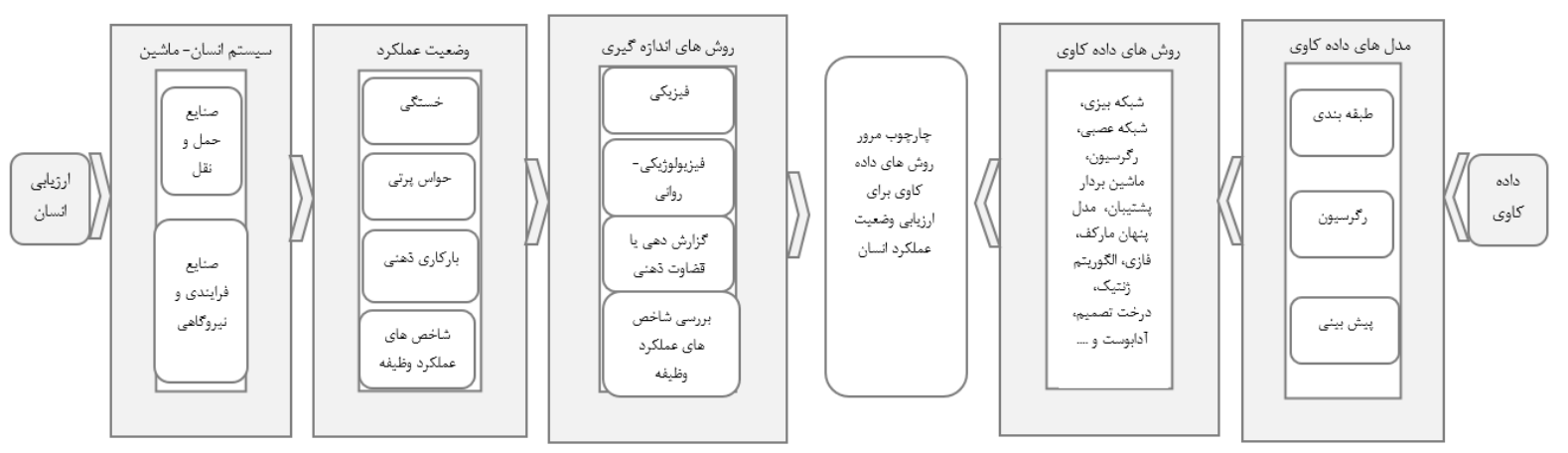

شكل ״ّ حار جوب سيستماتيك براى بررسى روش هاى داده كاوى در سيستم هاى انسان ماشين

امتيازدهى صحيح و بررسى دقيق تر، اين موارد به طور

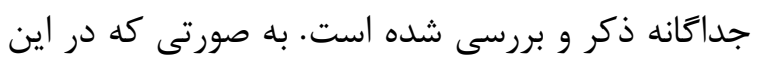

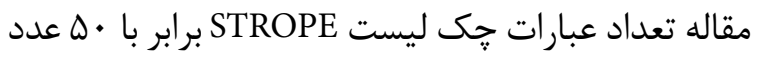

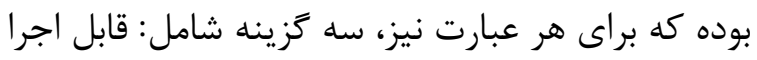

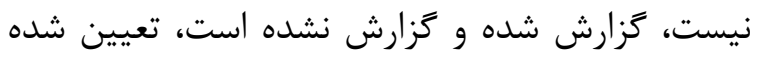

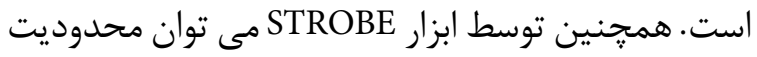

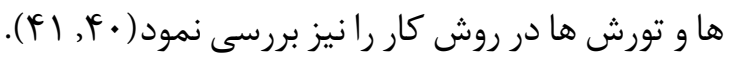

يافته ها

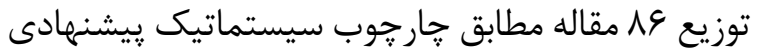
در جدول الرائه شده است.

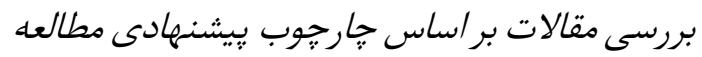

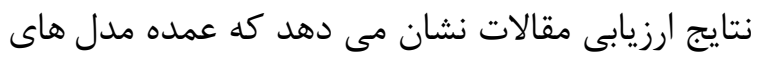

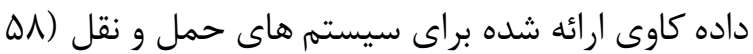

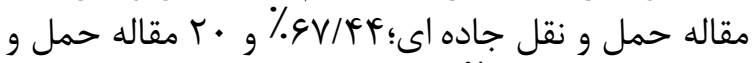

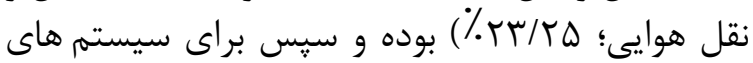

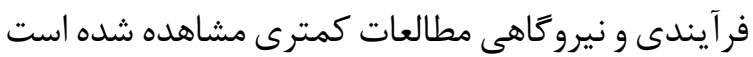

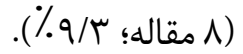

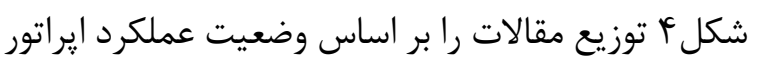

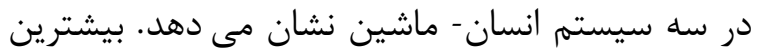

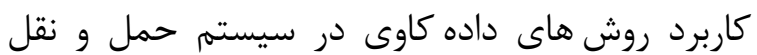

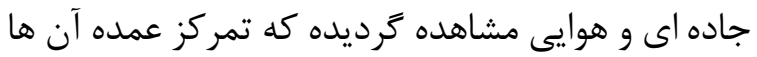

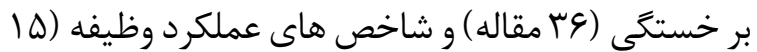

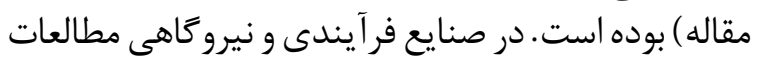

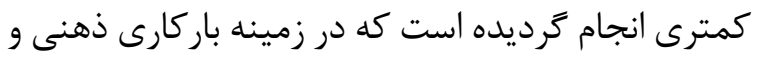

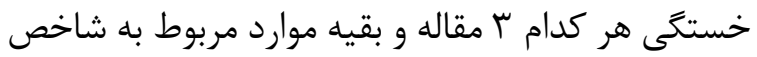

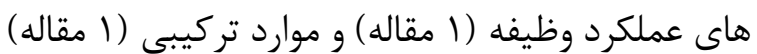

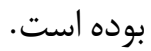
شكل ه كاربرد روش هاى اندازه گيرى را براى رينج گروه وضعيت عملكرد ايراتور نشان مى دهائ انداز كيرى راس اساس نتايج،

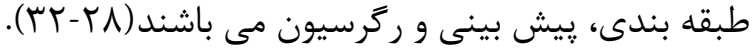

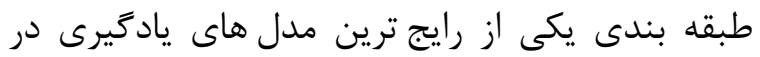

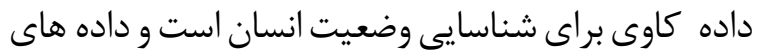

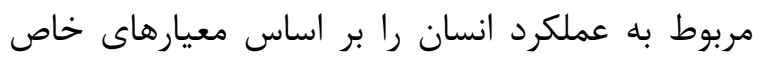

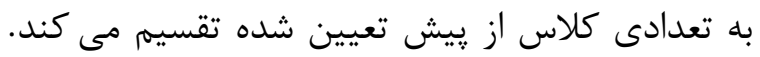

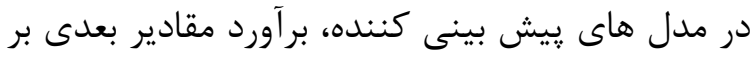

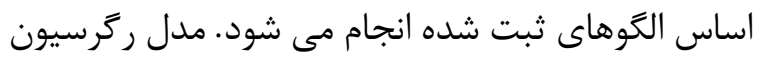

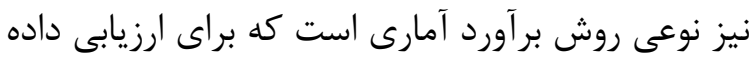

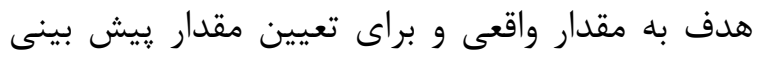

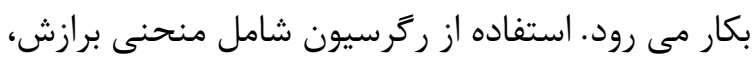

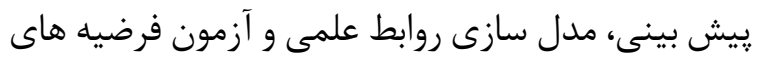

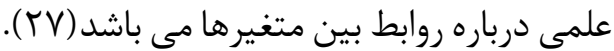

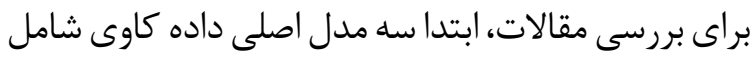

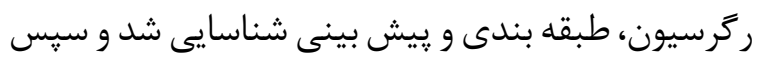

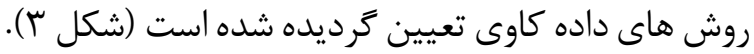

$$
\text { |رزيابى كيفيت مطالعات }
$$

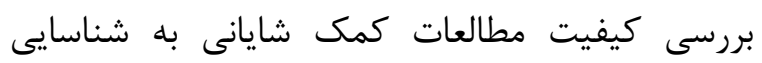

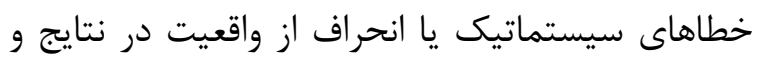

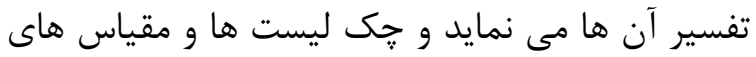

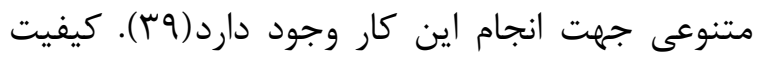

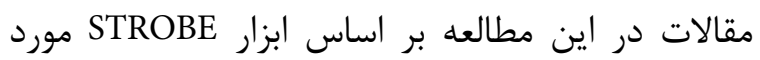

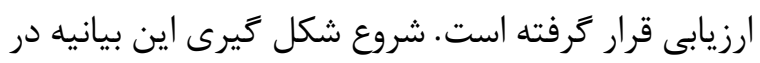

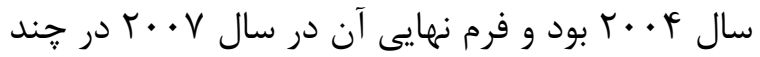

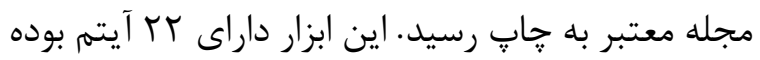

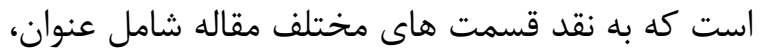

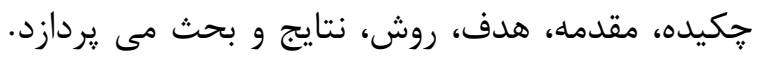

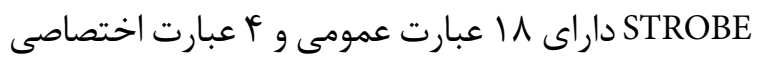

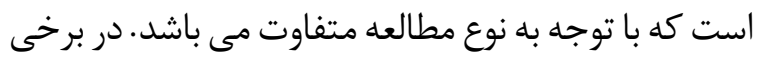

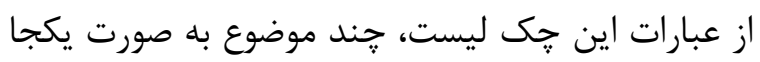
بيان شده اند كه بر اساس مطالعات بيشين وجند موض به مورت منظور 


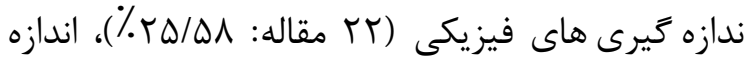
مدل هاى مربوط به يار امتر خستكى سهم بالاترى رانسبت

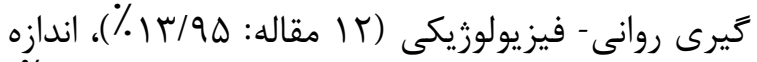

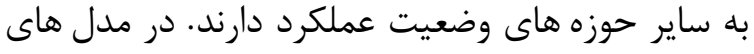

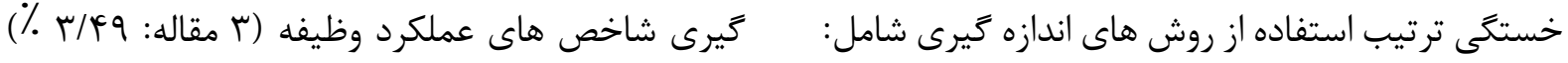

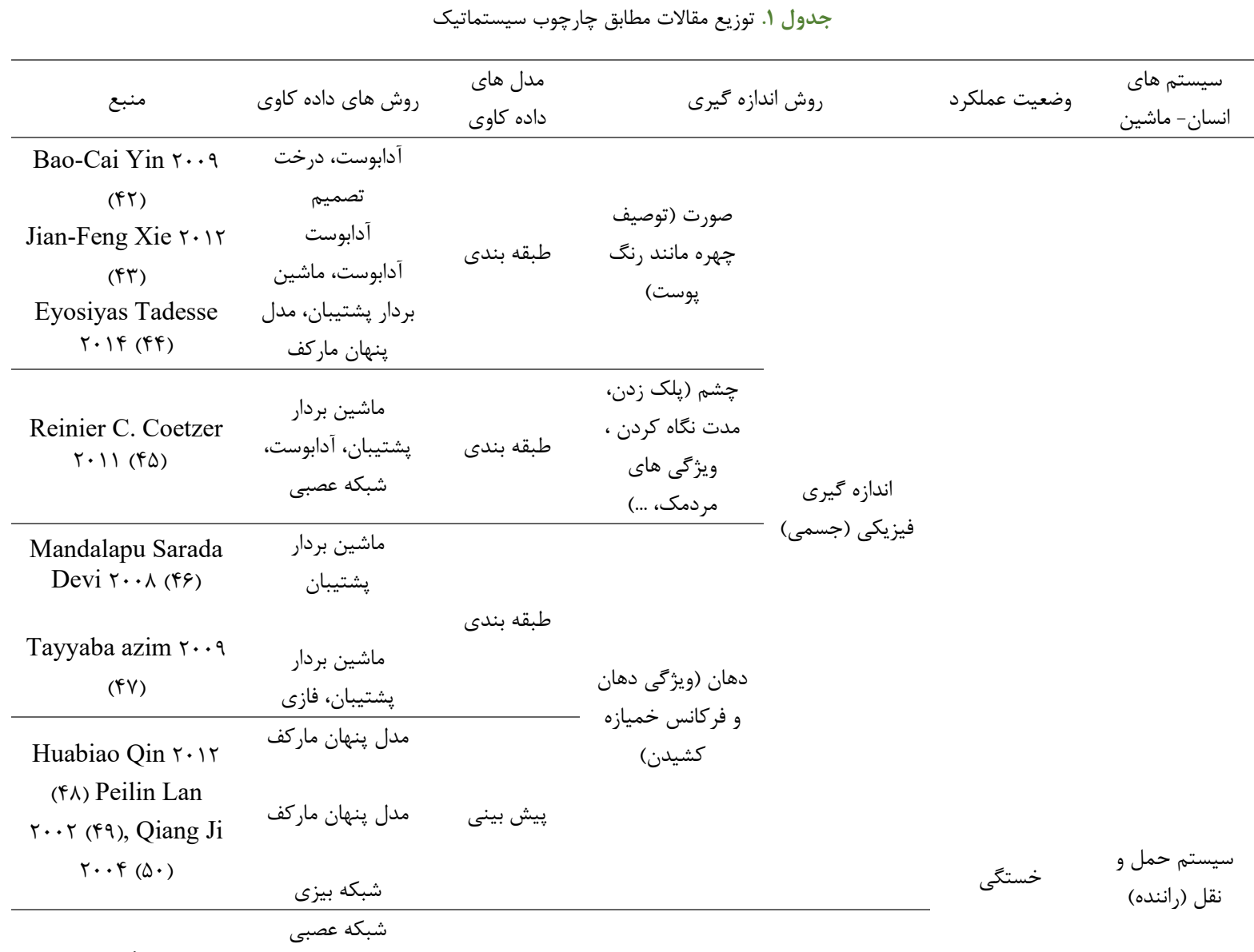

L. M. Kingr..ฯ

(DI)

شبكه عصبى

Mahesh M.

Bundele r...9 (Dr) ماشين بردار (QT),

$$
\begin{aligned}
& \text { الكتروانسفالوكرافى، } \\
& \text { الكتروكارديوكرافى، الكتركي، } \\
& \text { الكتروميوكرافى، اكتئ، } \\
& \text { اندازه كيرى روانى الكترواكولوكرافى، } \\
& \text { فعاليت الكتريكى } \\
& \text { فيزيولوزيكى }
\end{aligned}
$$$$
\text { Saroj Lal } r \cdots r
$$

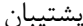$$
(\Delta \Delta) \text {, Issey }
$$

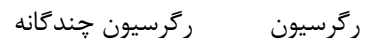

Takahashir. 11

$$
(\Delta \&)
$$

Zhiwei Zhu r.. $\mathrm{f}$

$$
\text { شبكه بيزى }
$$

Mitesh Patel $r \cdot 11$

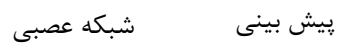

Rami Khushaba

$$
r \cdot 1 \cdot(\Delta q)
$$

$$
\text { فازى }
$$




\begin{tabular}{|c|c|c|c|c|c|c|}
\hline \multicolumn{7}{|c|}{ ادامه جدول ا. توزيع مقالات مطابق خار خوب سيستماتيك } \\
\hline منبع & داده كاوى هاى & مدل هاده كاوى & 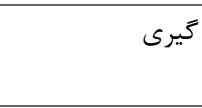 & 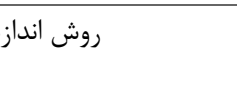 & وضعيت عملكرد & سيستم \\
\hline $\begin{array}{c}\text { Youngjae Kim } \\
r \cdot \wedge(q \cdot) \\
\text { Esra Vural } \\
r \cdots \vee, r \cdots q, \xi)) \\
(q r\end{array}$ & 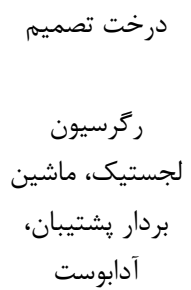 & طبقه بندى & زمان زينَين سرعت و & هاى عملكرد وظيفه شاخص & & \\
\hline $\begin{array}{l}\text { Wang Rang- } \\
\text { ben } r \cdot \cdot r(\mathcal{G})\end{array}$ & شبكه عصبى & & & & & \\
\hline $\begin{array}{l}\text { Xiao Fan } r \cdots \wedge \\
(\notin \uparrow), \text { Ping }\end{array}$ & شبكه بيزى & & & & & \\
\hline $\begin{array}{l}\text { Wang r.Ir } \\
(\xi \Delta), \text { Xiao-qing }\end{array}$ & آدابوست & & & & & \\
\hline Luo r. Ir (\$9) & آدابوست & & & & & \\
\hline $\begin{array}{l}\text { Chao Sun } r \cdot l^{r} \\
(q \vee) \text { Madhuri R. }\end{array}$ & آدابوست & & & & & نقل (راننده) حمل و \\
\hline $\begin{array}{c}\text { Tayade } r \cdot \mid l^{q} \\
(\xi \wedge) \\
\text { Rajinda }\end{array}$ & ماشين بردار & 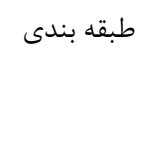 & & & & \\
\hline $\begin{array}{c}\text { Senaratner } \cdots v \\
\text { (६9) }\end{array}$ & ماشين بردار & & (رديابى هشه و خشم، & & & \\
\hline $\begin{array}{c}\text { Jian-Da Wu } \\
\vdash \cdot \wedge(\vee \cdot)\end{array}$ & فازى & & 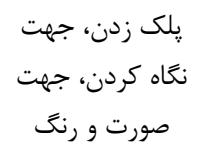 & اندازه كيرى فيزيكى & خستكى & \\
\hline $\begin{array}{l}\text { A. M. Bagci } \\
r \ldots r(V))\end{array}$ & مدل ينهان & & ) يوست) & & & \\
\hline $\begin{array}{c}\text { Shuyan } \mathrm{Hu} \\
r .9(V Y) \\
\text { Thomas Brandt } \\
r . . r(V r)\end{array}$ & ماشين بردار & 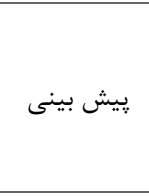 & & & & \\
\hline $\begin{array}{c}\text { Shanshan Jin } \\
r \cdot V(V \mathcal{F})\end{array}$ & شبكه عصبى زلكَيك، & پِيش بينى & 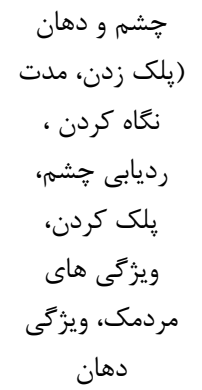 & & & \\
\hline $\begin{array}{l}\text { Ralph Oyini } \\
\text { Mbouna } r \cdot 1 r \\
(\vee \Delta)\end{array}$ & ماشين بردار & 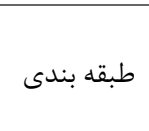 & جشم و زاويه سر & & & \\
\hline
\end{tabular}




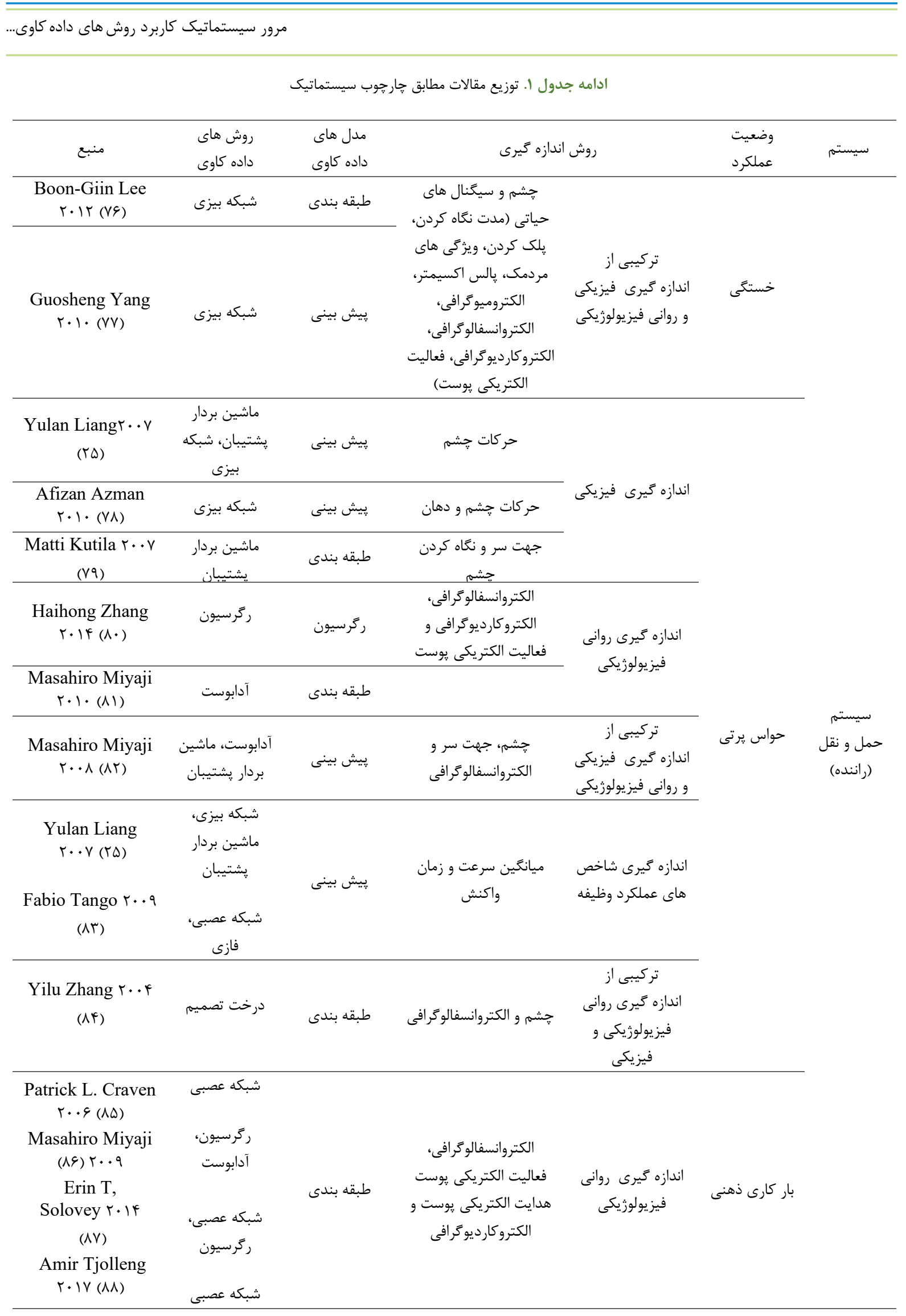


ادامه جدول ا. توزيع مقالات مطابق خارحوب سيستماتيك

\begin{tabular}{|c|c|c|c|c|c|c|}
\hline منبع & راده كاوى هاى & مدل هاده كاوى & \multicolumn{2}{|c|}{ روش اندازه گيرى } & وضعيت عملكرد & سيستم \\
\hline $\begin{array}{c}\text { Wenhui Liao } \\
\ulcorner. . \Delta(\wedge 9)\end{array}$ & شبكه بيزى & ي يـش بينى & 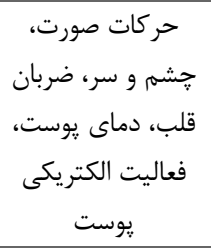 & كَيرى فيزيكى و ورواندازه & بار كارى ذهنى & \\
\hline $\begin{array}{c}\text { Jasper Wijnands } \\
\uparrow \cdot \backslash \wedge(q \cdot)\end{array}$ & شبكه عصبى & پِيش بينى & كاهش و افزايش سرعت & هاى عملكرد وظيفه شاخص & شاخص هاى عملكرد & \\
\hline
\end{tabular}

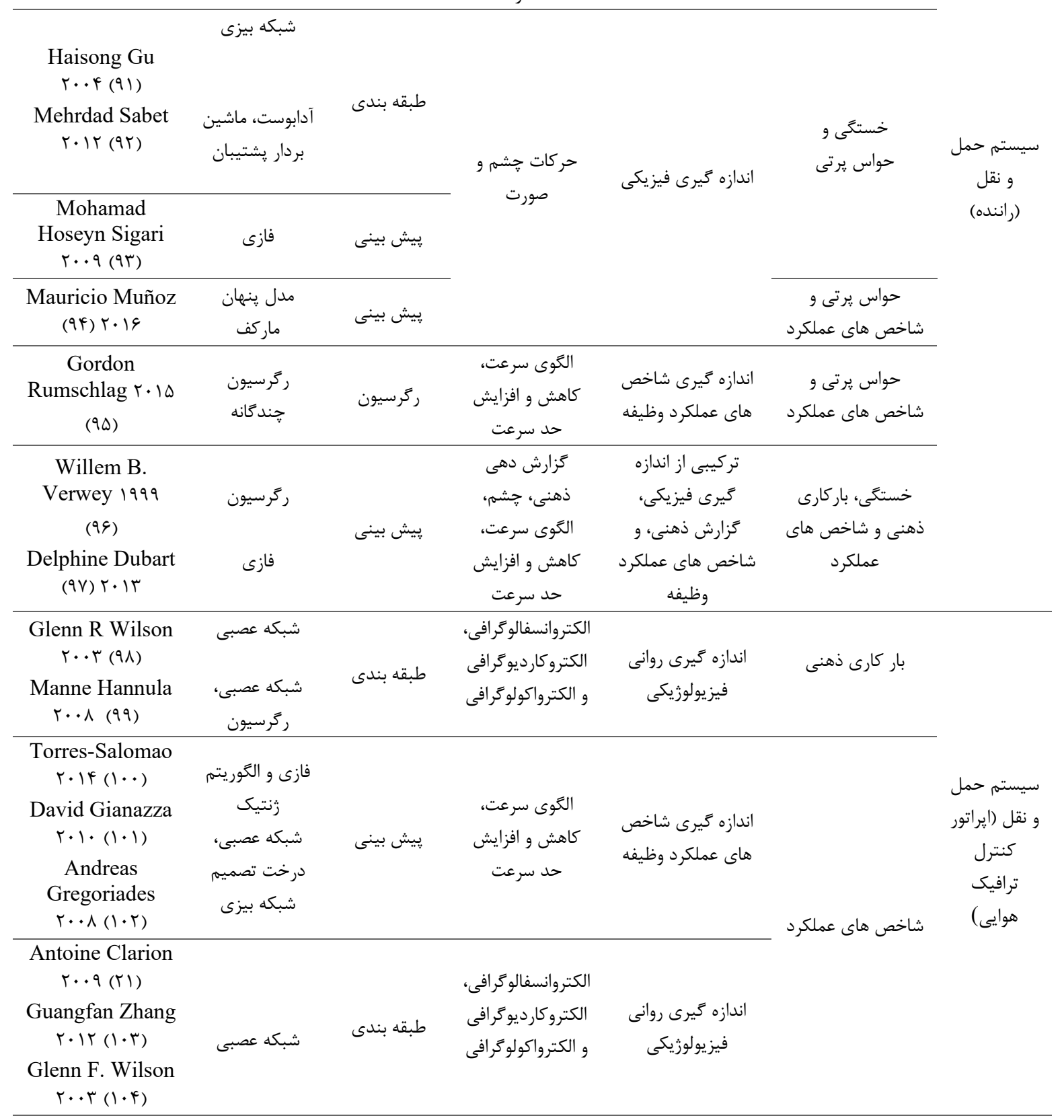




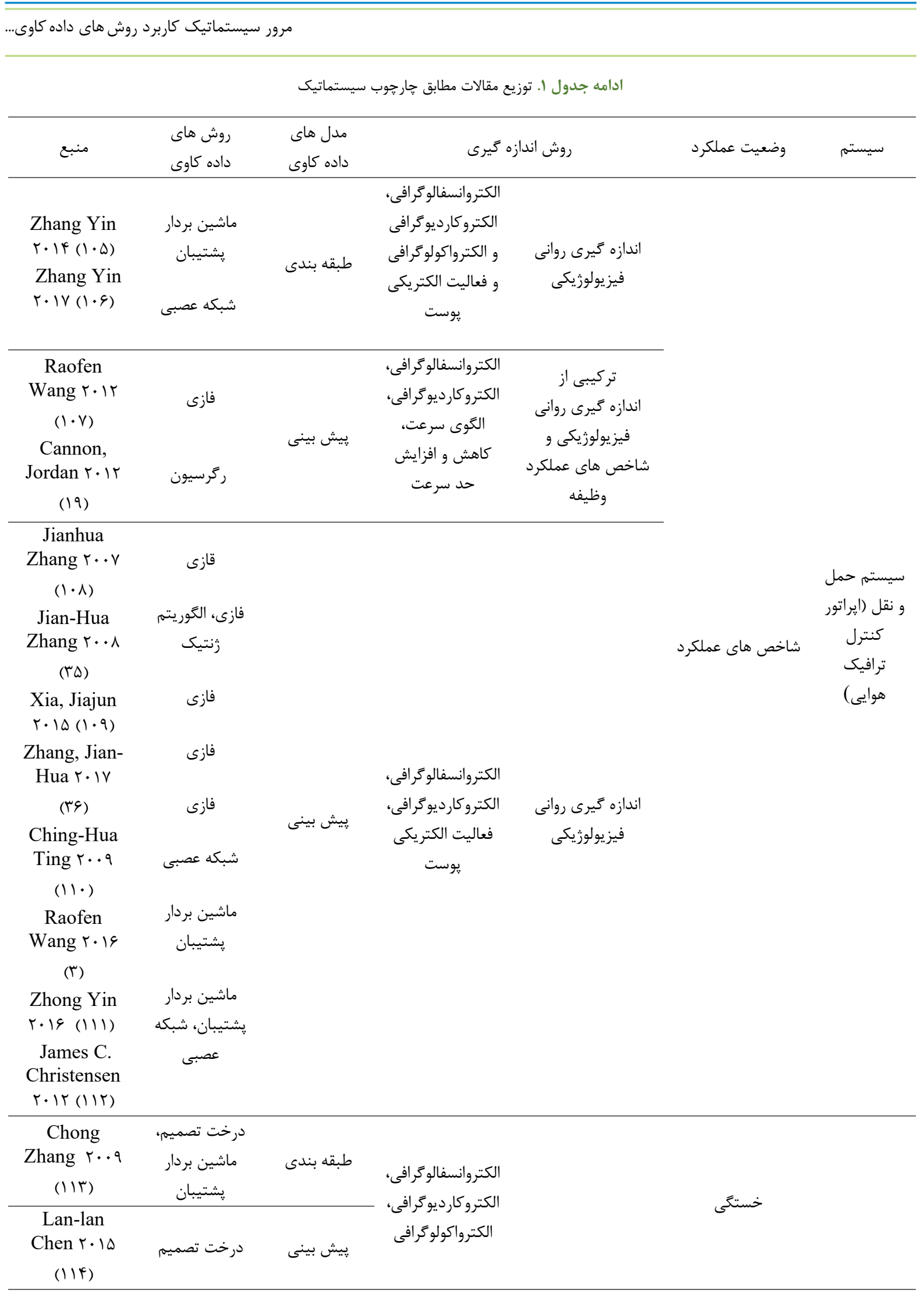


ادامه جدول ا. توزيع مقالات مطابق خار خوب سيستماتيك

\begin{tabular}{|c|c|c|c|c|c|c|}
\hline منبع & داده كاوى هاى & مدل هاد كاوى & & روش اندازه گيرى & وضعيت عملكرد & سيستم هاى انسان ماشين \\
\hline $\begin{array}{c}\text { François } \\
\text { Laurent } r \cdot \mid r \\
((1 \otimes)\end{array}$ & ماشين بردار & طبقه بندى & الكتروكارديوگر افىى، الكتراكي، & فيزيولوزيكىى روانى & خستگى & \\
\hline $\begin{array}{l}\text { B.S. Moon } \\
r . r(119)\end{array}$ & فازى & & & & & \\
\hline $\begin{array}{l}\text { Rouja Nikolova } \\
r \cdot r \text { ( } \mid \backslash), \\
\text { Yufeng Ke } \\
r \cdot \mid \uparrow(\mid \backslash \Lambda)\end{array}$ & ماشين برديون & طبقه بندى & الكتروانسفالوكرافى، الكتردئ & فيزيولوزيكيكى روانى & بار كارى ذهنى & \\
\hline $\begin{array}{l}\text { Hwang, Sheue- } \\
\text { Ling } r \cdots \wedge(r \vee)\end{array}$ & شبكه عصبى & پِيش بينى & 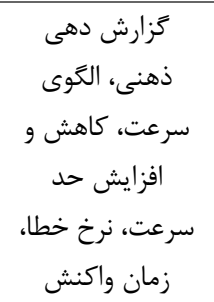 & عَ عزيرى شاخصى از اندازه & شاخص هاى & فريستمًا سيندى و \\
\hline $\begin{array}{l}\text { Hwang, Sheue- } \\
\text { Ling } r . .9(Y Y)\end{array}$ & فازى & پِيش بينى & 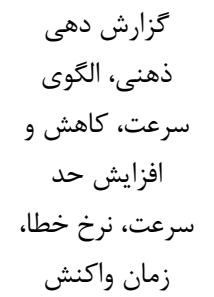 & 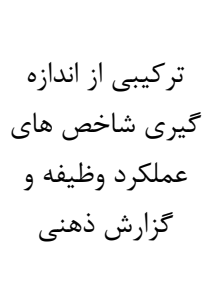 & شاركارى ذهنى و هاخ & \\
\hline
\end{tabular}

رده هاى بعدى قرار زرفته اند.

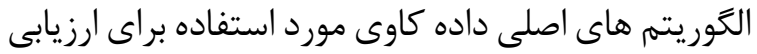

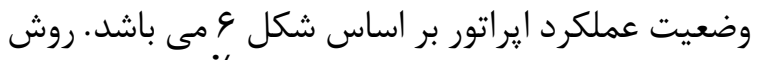

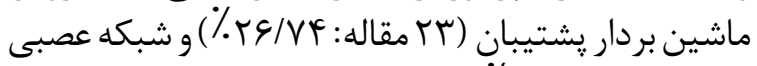

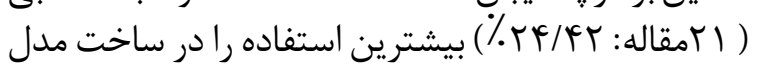

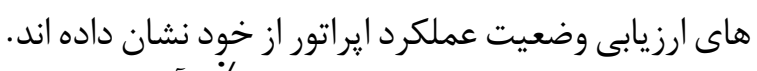

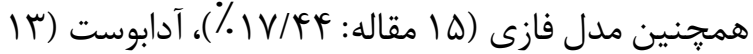

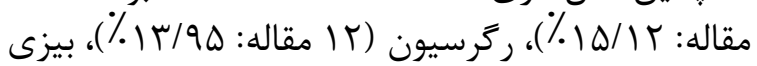

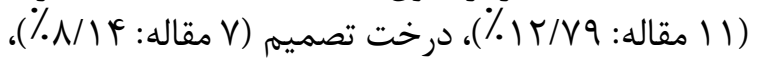

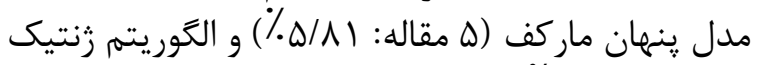

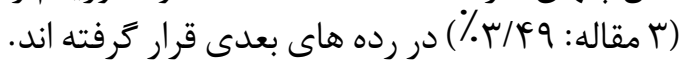

$$
\text { توزيع مقالات بر /ساس سال انتشار }
$$

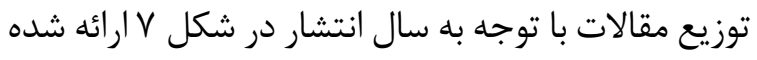

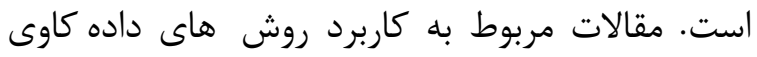

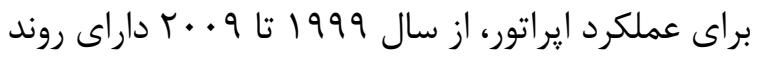

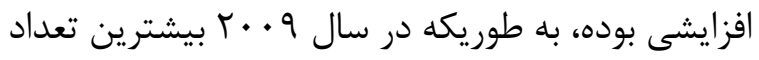

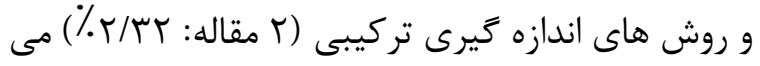

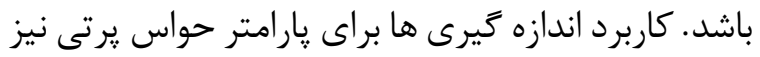
به صورت روش هاى اندازه گيرى فيزيكى شامل با مقاله

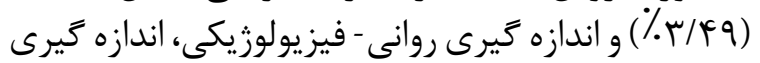
شاخص هاى عملكرد وظيفه و روش هاى اندازه كيرى أنى

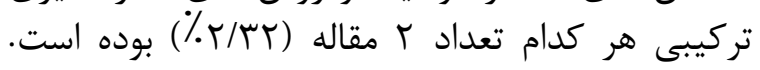
روش هاى اندازه گيرى روانى - فيزيولوزيكى بيشى نيشترين كاربرد را براى ساخت مدل هاى بار كارى ذهنى ذهى و شاخد

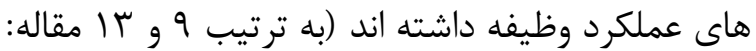

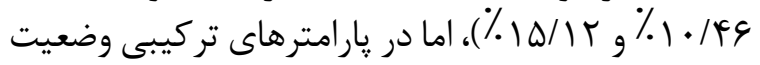
عملكرد، از روش هاى اندازه خيرى فيزيكى بيشترئ

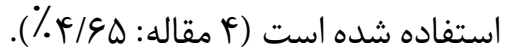

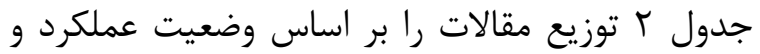

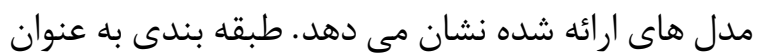

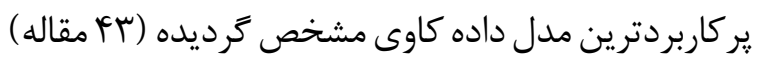

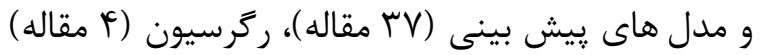
و همجنين تركيبى از مدل هاى داده كاوى (ץ مقاله) در 
مرور سيستماتيك كاربرد روش هاى داده كاوى...

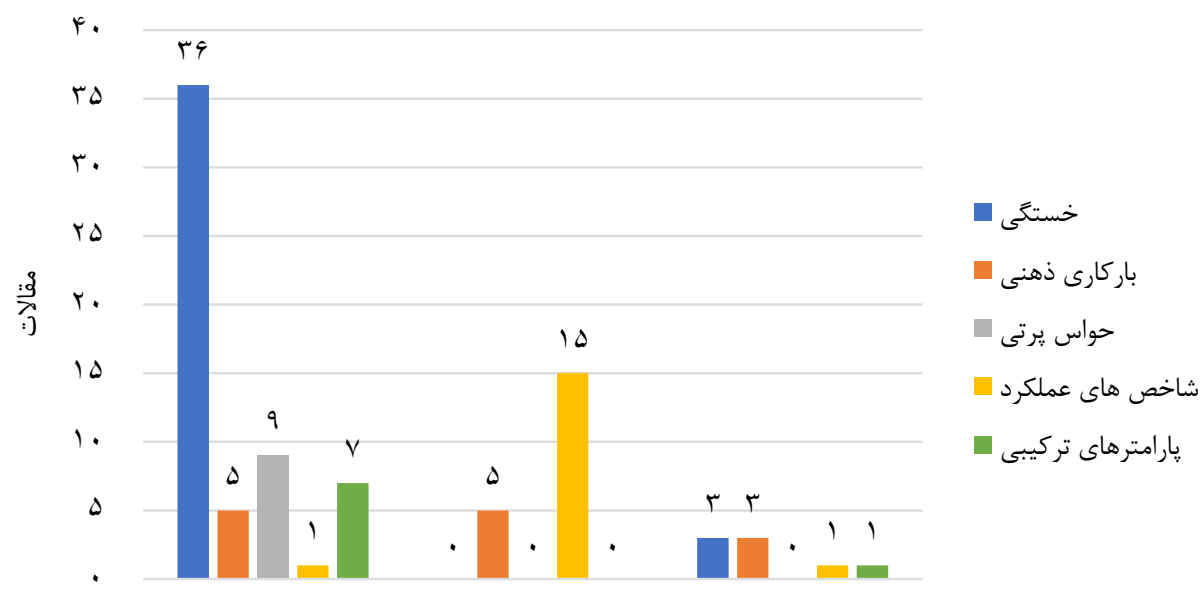

سيستم فرايندى و تيروكاهى سيستم حمل و نقل هوايى سيستم حمل و نقل جاده اى

شكل Fا. توزيع مقالات بر اساس ارزيابى وضعيت عملكرد ايراتور در سيستم هاى انسان- ماشين مورد بررسى

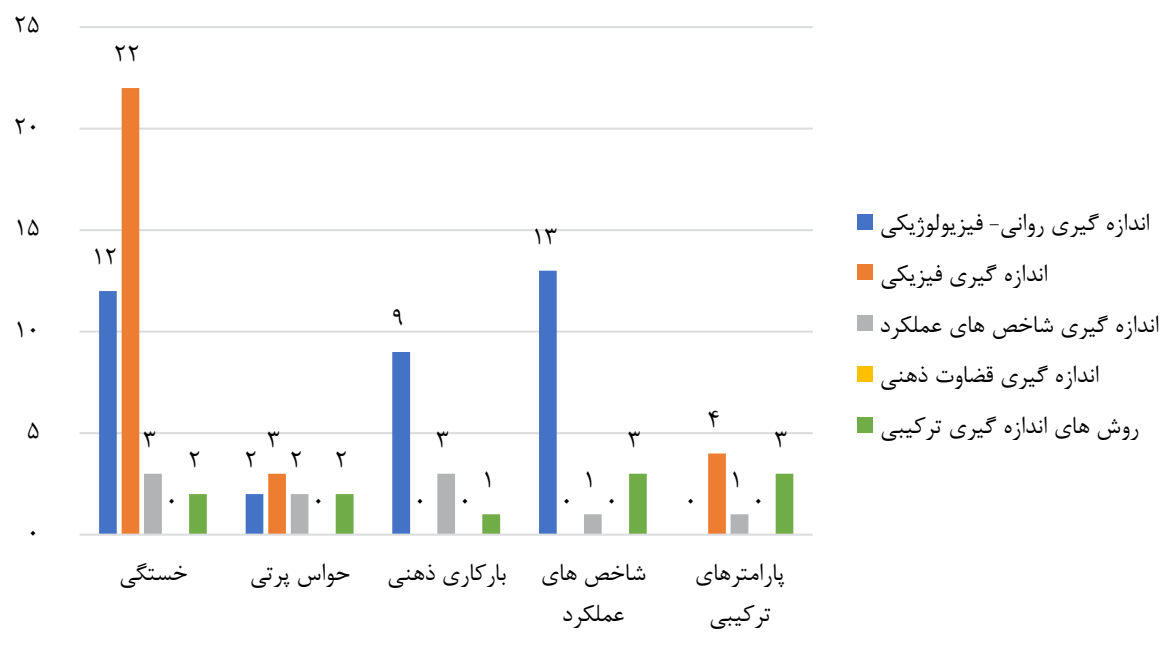

شكل ه. توزيع مقالات بر اساس روش هاى اندازه گيرى وضعيت عملكرد ايراتور

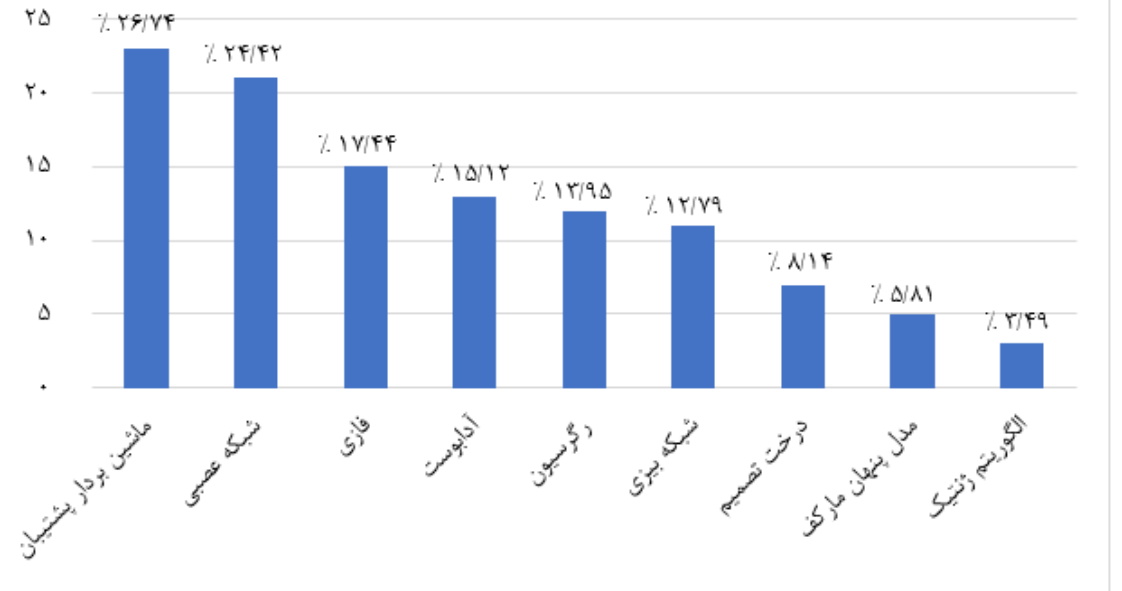

شكل 9. توزيع مقالات بر اساس كاربرد روش هاى داده كاوى 


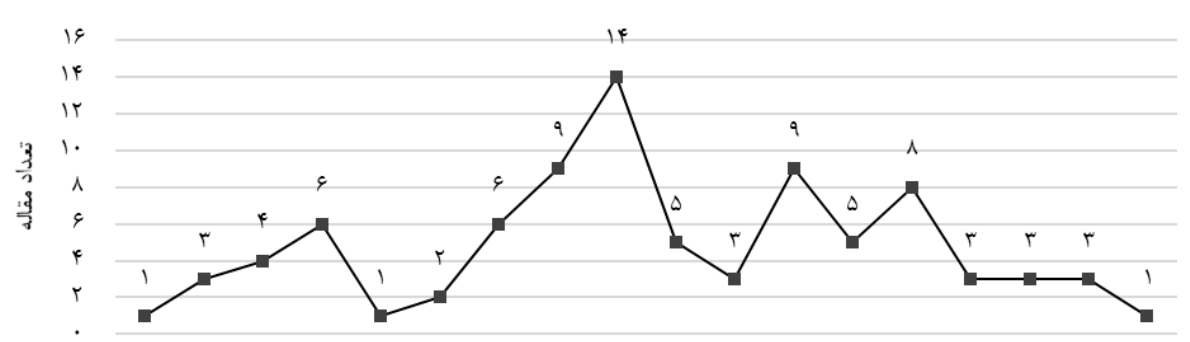

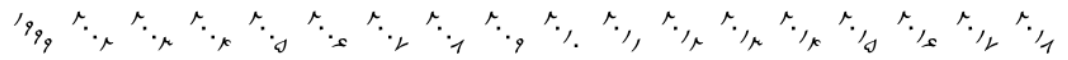

$$
\begin{aligned}
& \text { سال جاب }
\end{aligned}
$$

شكل V. توزيع مقالات بر اساس سال انتشار

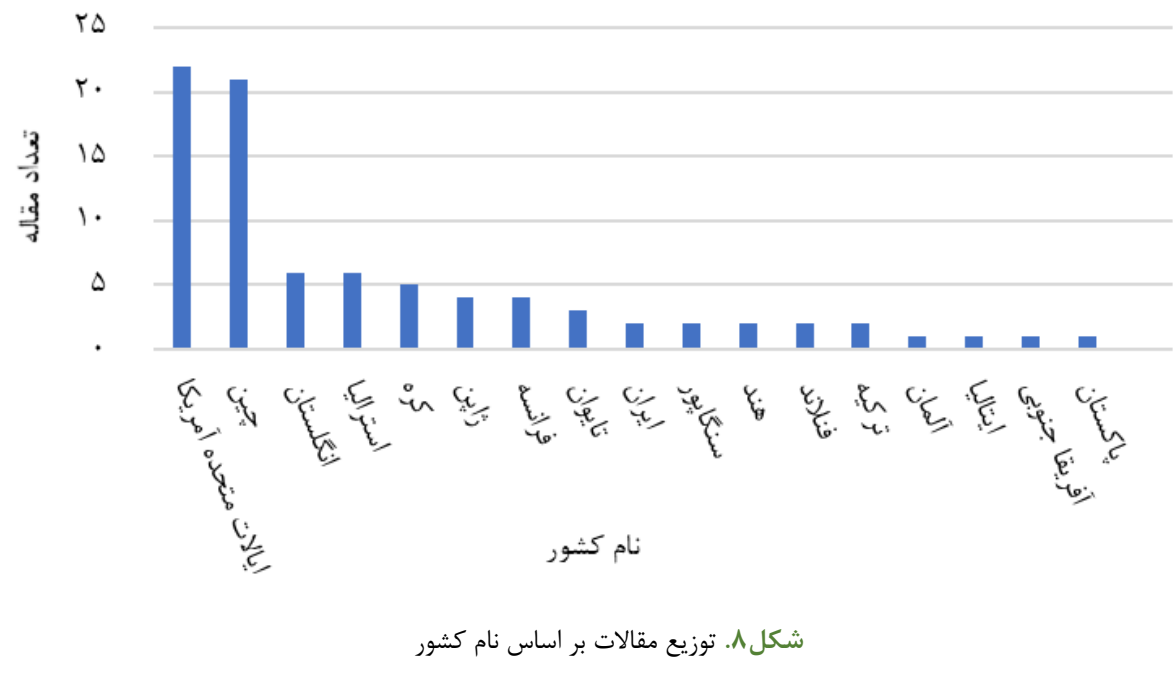

مقاله در اين حوزه منتشر شده است. يس از سال 9 ـ . ؟؟،

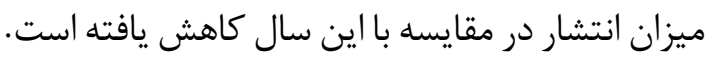

$$
\text { توزيع مقالات بر اساس نام كشور }
$$

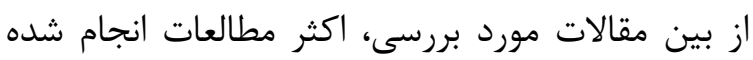

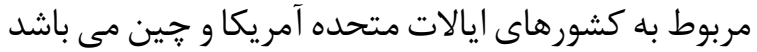

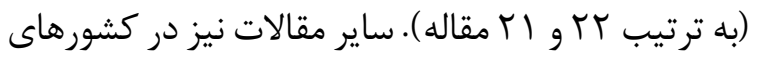

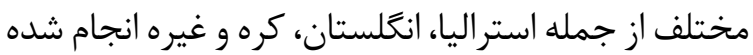

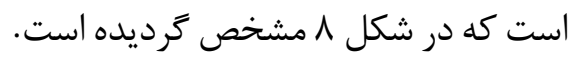

بحث مهثى از هر مطالعه مرورى سيستماتيكي، ارائه يك بخش مهمى از هر مطالعه مرورى سيستماتيك، ارائه يك

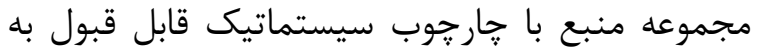

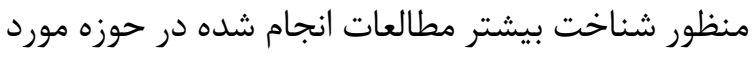

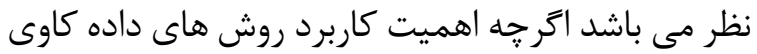

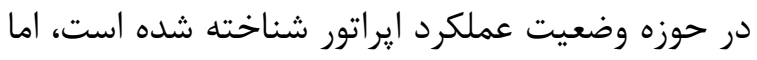
ارزيابى جامعى از حوزه هاى مورد توجه، روش هاى مورد إندان
جدول r. توزيع مقالات بر اساس وضعيت عملكرد إِراتور و مدل هاى

\begin{tabular}{|c|c|c|}
\hline ت تعداد مقاله & مدل هاى داده كاوى & وضعيت عملكرد ايراتور \\
\hline TY & طبقه بندى & \\
\hline 11 & بيش بينى & خستح \\
\hline r & ر ركرسيون & 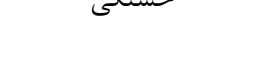 \\
\hline r & تركيبى & \\
\hline r & طبقه بندى & \\
\hline$\Delta$ & رِيش بينى & حواس يرتى \\
\hline 1 & ركرسيون & \\
\hline 9 & طبقه بندى & الا كا، ذهن ذهن \\
\hline r & ي ييش بينى & بر مارى دهى \\
\hline$\Delta$ & طبقه بندى & شاخص هاي عملكرد \\
\hline ir & بيش بينى & \\
\hline r & طبقه بندى & \\
\hline$\Delta$ & بيش بينى & يارارترهاى تركيبى \\
\hline 1 & رَر سيون & \\
\hline 19 & كل & كل \\
\hline
\end{tabular}
داده كاوى 
تكنيك هاى داده كاوى مورد استفاده در تعيين وضعيت

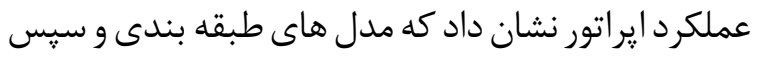

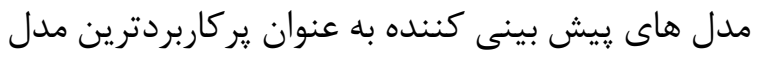

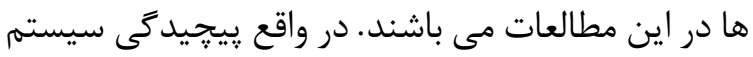

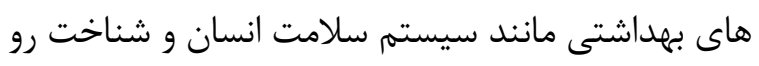
به رشد آن ها و همجنين نياز به ماند سياست كذارى و و تصميم

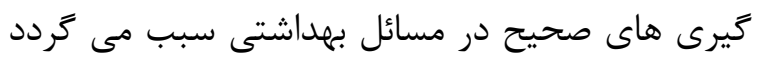

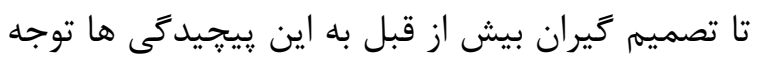

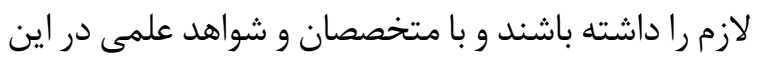

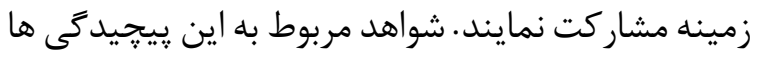

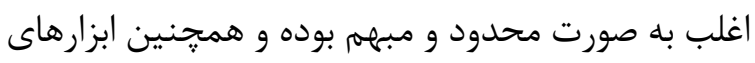

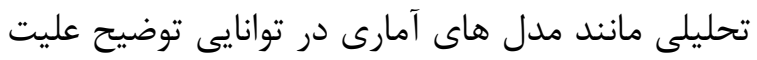

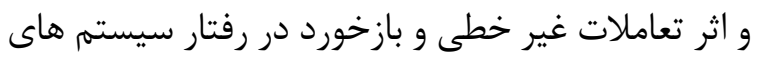

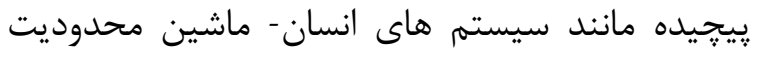

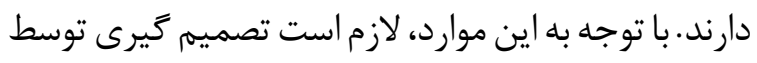

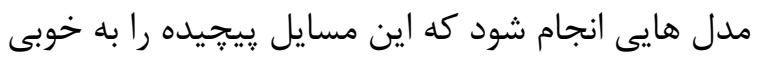

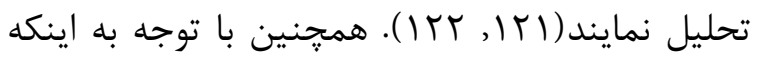

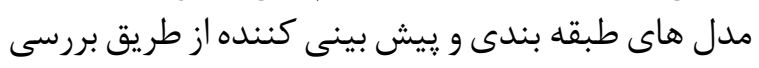

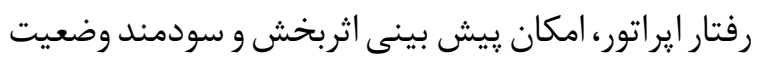

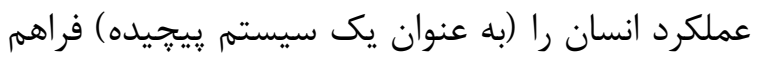

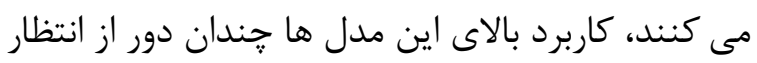

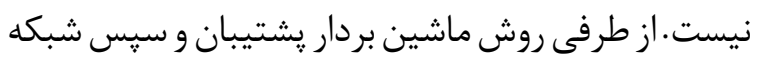

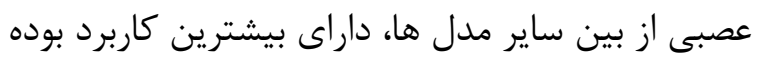

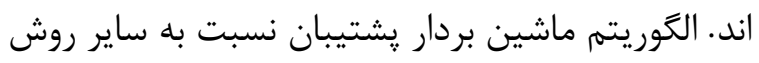

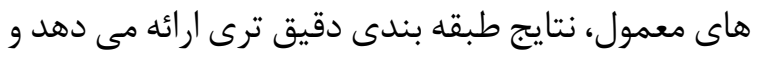

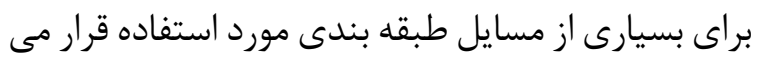

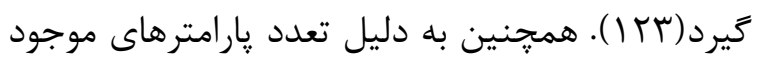

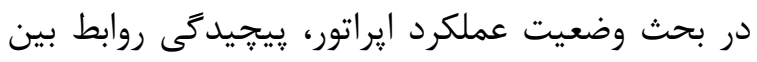

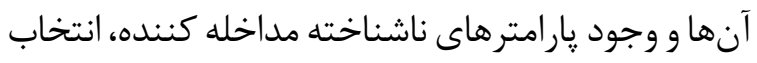

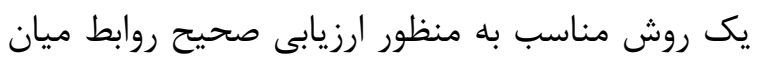

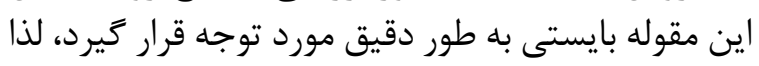

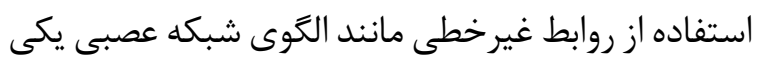

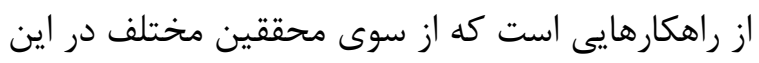

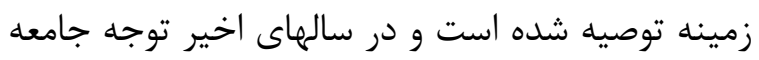

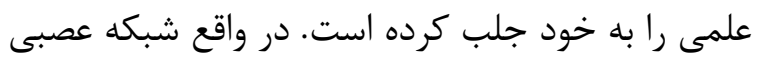

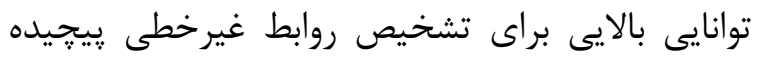

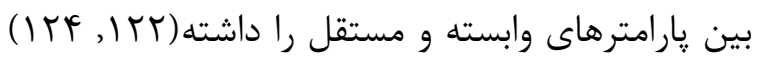

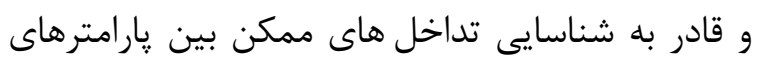

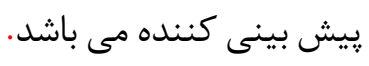
همجنين مدلسازى توسط بـ روش هاشد هاى آمارى كلاسيك،

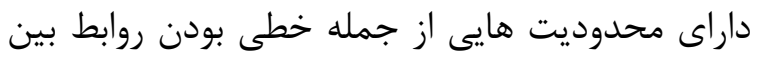

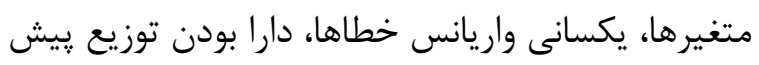

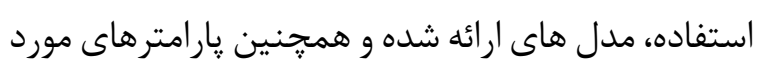

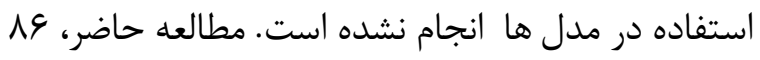

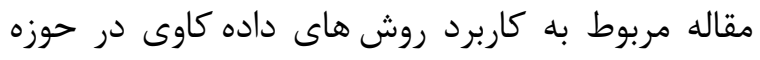

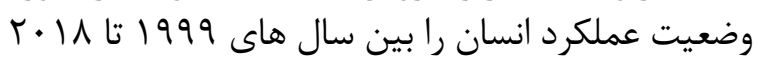

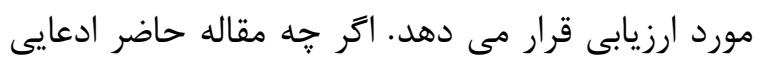

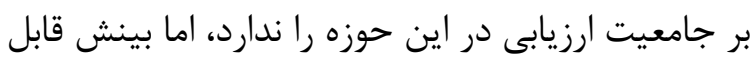

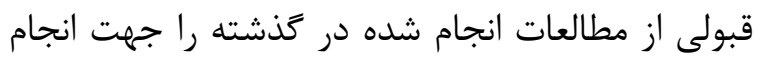
مطالعات جديد ارائه مي نمايد.

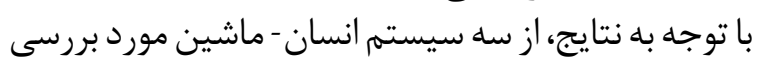

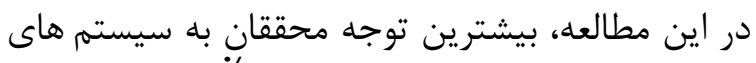

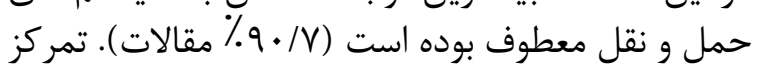

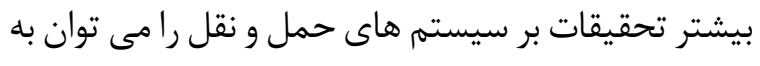

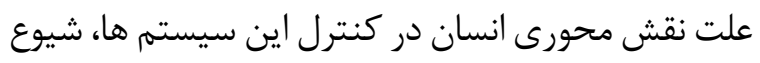

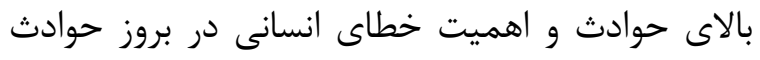

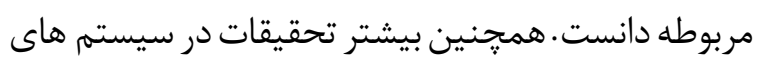

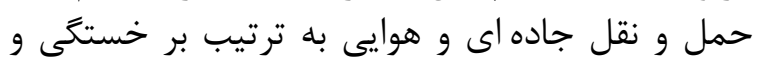

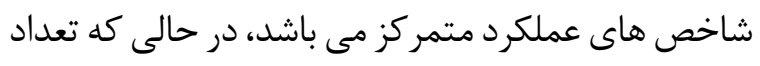

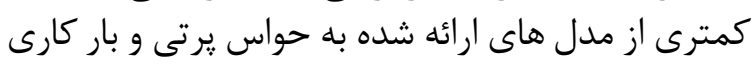

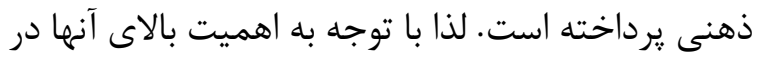

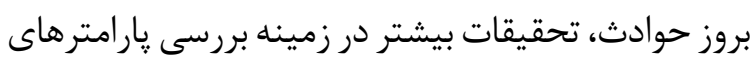

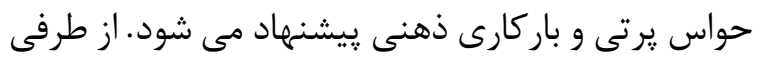

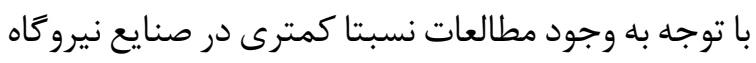

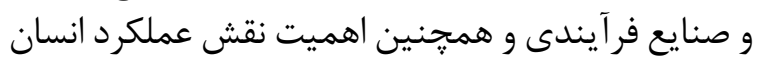

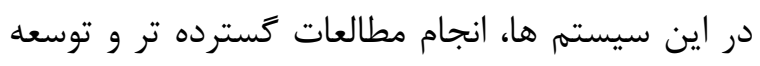

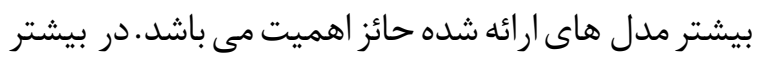

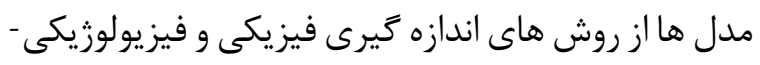

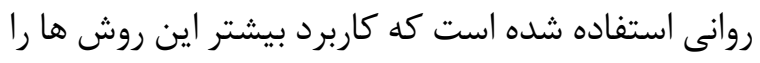

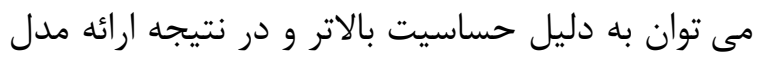

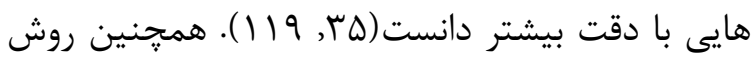

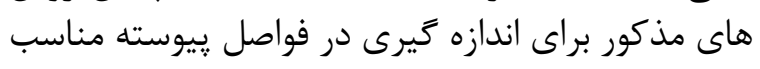

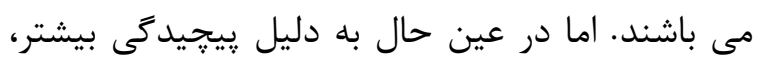

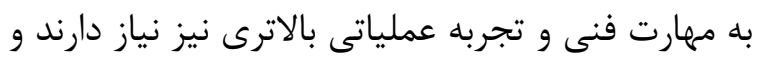

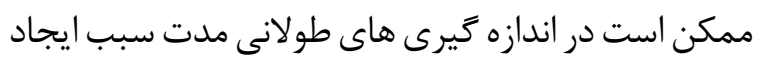

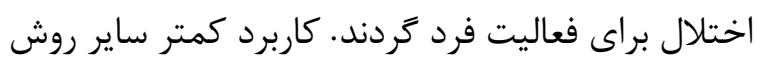

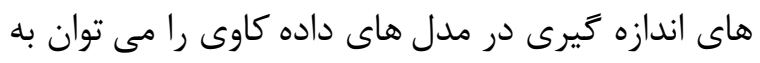

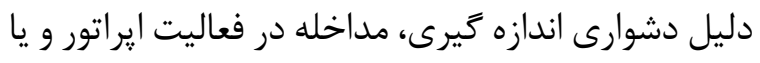

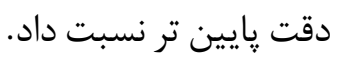

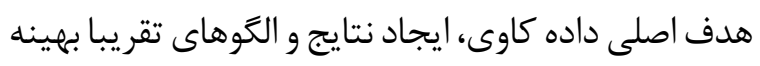

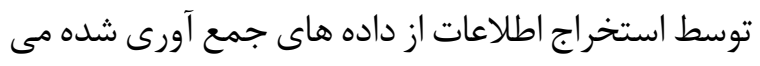

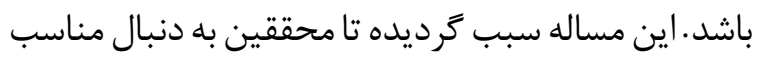

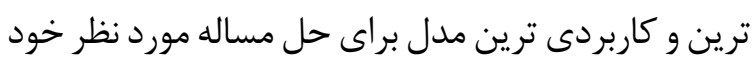

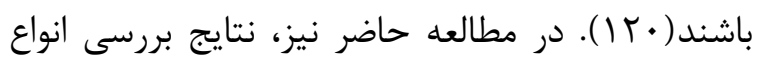




\section{نتيجه كَيرى}

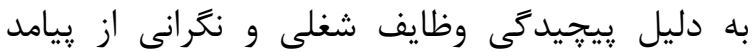

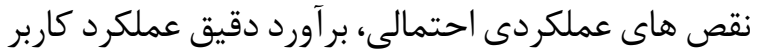

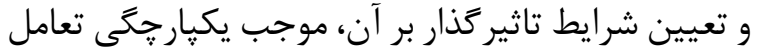

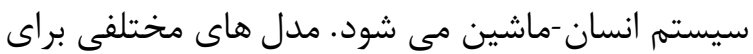

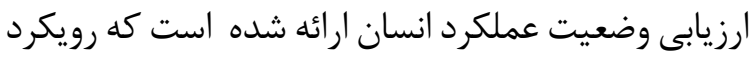
كلى اين مدل ها، توجه به بِيجيديد

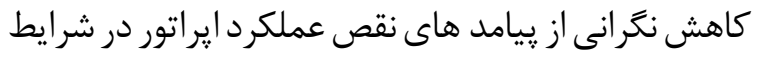

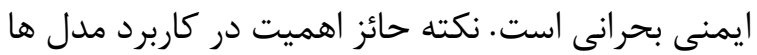

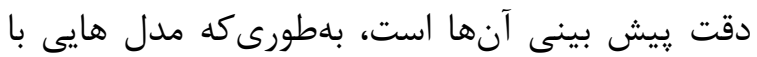

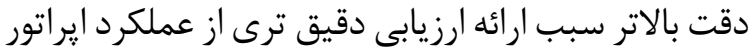

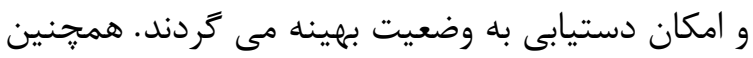

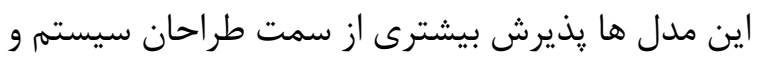

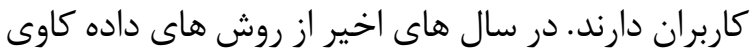

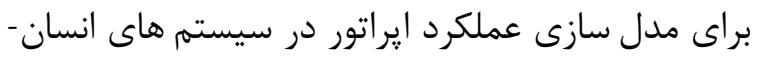
ماشين به فراوانى استفاده شده است كه كه روش ماري ماشين

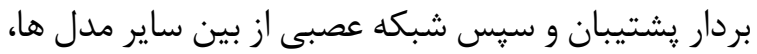

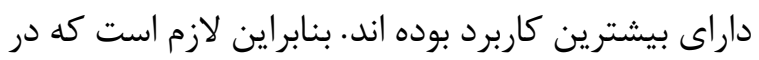

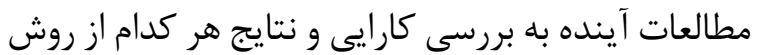

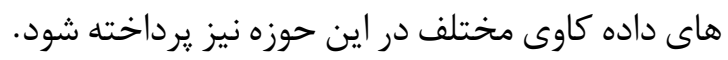

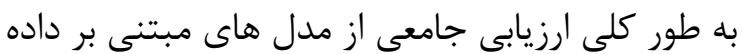

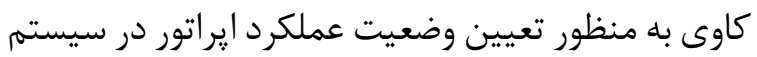

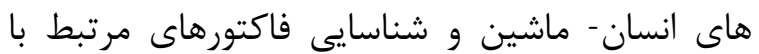

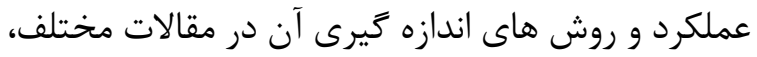

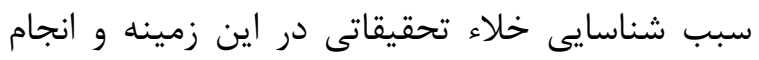

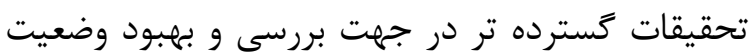

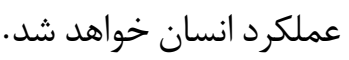

1.Ghaneh S, Lefti A. Occupational ergonomics, Principles and applications. Tehran: Fadak isatis. (Persian).

2.Golmohammadi R, Motamedzade M, Mahdavi N. Occupational Ergonomics. Hamedan: Daneshjoo; 2017. (Persian)

3.Wang R, Zhang Y, Zhang L. An adaptive neural network approach for operator functional state prediction using psychophysiological data. Integrated Computer-Aided Engineering. 2016;23(1):81-97.

4.Hockey GRJ. Operator functional state: the assessment and prediction of human performance degradation in complex tasks: IOS Press; 2003.

5.Papantoniou P, Papadimitriou E, Yannis G. Review of driving performance parameters critical for distracted driving research. Transportation research procedia.
فرض براى متغيرها و غيره مى باشند و قابليت مدلسازى

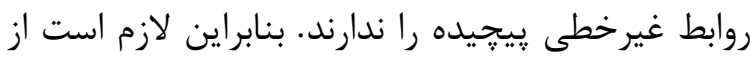

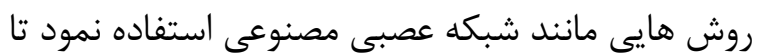

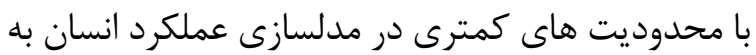

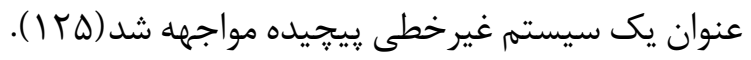

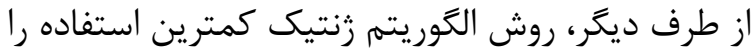

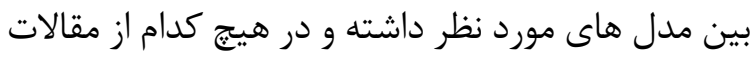

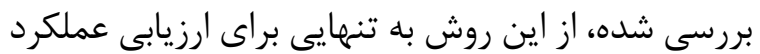

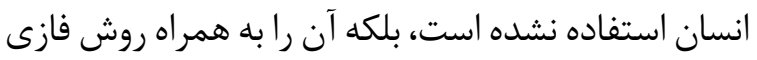

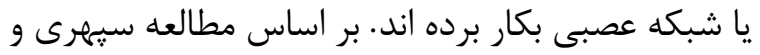

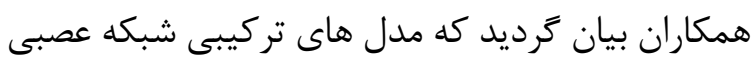

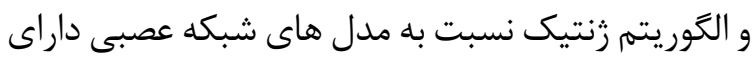

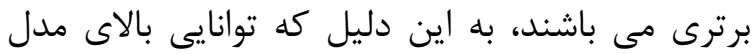

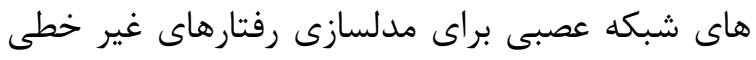

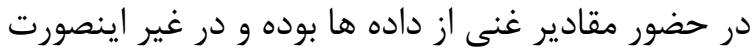

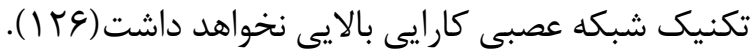

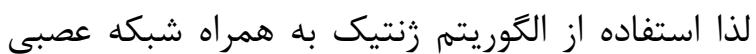

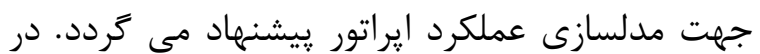

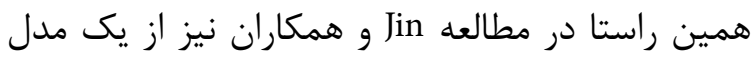

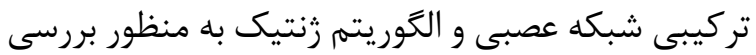

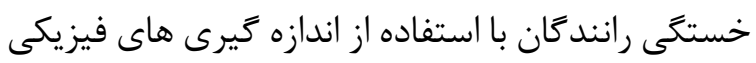

شامل حركات جشمه و دهان استفاده شده است (VY)

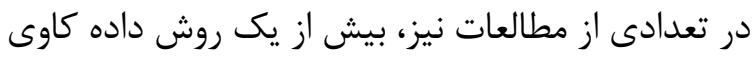

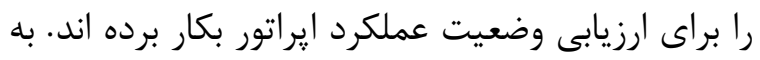

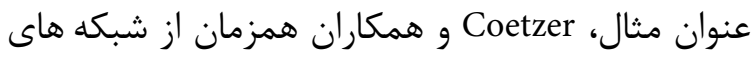

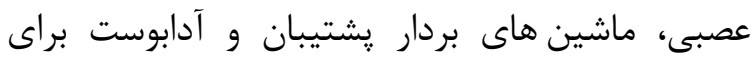

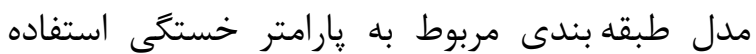

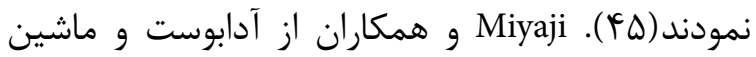

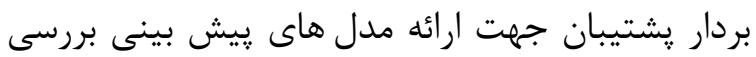

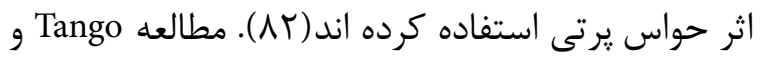

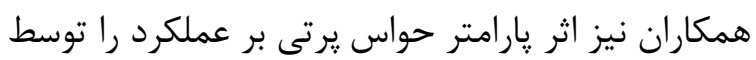

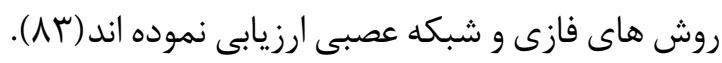

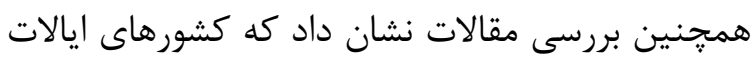

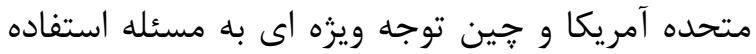

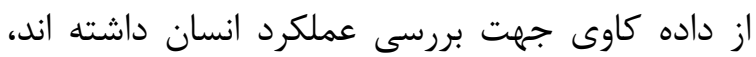

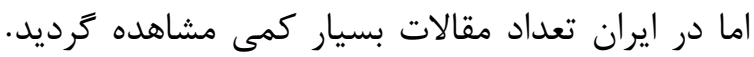

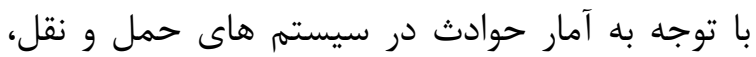

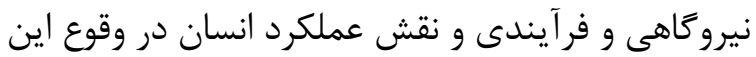

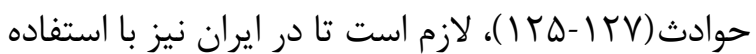

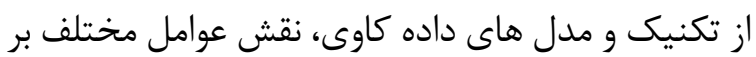

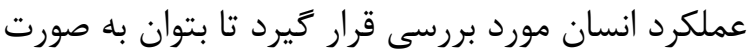
هدفمند نسبت به كنترل حوادث اقدام نمود. 
مرور سيستماتيك كاربرد روش هاى داده كاوى...

review of recent progress. Multiple-task performance. 1991:279-328.

21.Clarion A, Ramon C, Petit C, Dittmar A, Bourgeay J, Guillot A, et al. An integrated device to evaluate a driver's functional state. Behavior research methods. 2009;41(3):882-8.

22.Friswell R, Williamson A. Comparison of the fatigue experiences of short haul light and long distance heavy vehicle drivers. Safety science. 2013;57:203-13.

23.Wilson GF, Russell CA. Performance enhancement in an uninhabited air vehicle task using psychophysiologically determined adaptive aiding. Human factors. 2007;49(6):1005-18.

24.Hwang S-L, Liang G-F, Lin J-T, Yau Y-J, Yenn T-C, Hsu $\mathrm{C}-\mathrm{C}$, et al. A real-time warning model for teamwork performance and system safety in nuclear power plants. Safety science. 2009;47(3):425-35.

25.Liang Y, Lee JD, Reyes ML. Nonintrusive detection of driver cognitive distraction in real time using Bayesian networks. Transportation research record. 2007;2018(1):1-8.

26. Sadeghian M. Investigating cognitive performance under different Annoyance and mental workloadusing artificial neural network. PhD thesis. Shahid Beheshti University of Medical Sciences, School of Public Health and Safety; 2019. (Persian).

27.Nisbet R, Elder J, Miner G. Handbook of statistical analysis and data mining applications: Academic Press; 2009.

28.Kiymik MK, Akin M, Subasi A. Automatic recognition of alertness level by using wavelet transform and artificial neural network. Journal of neuroscience methods. 2004;139(2):231-40.

29.Yildiz A, Akin M, Poyraz M, Kirbas G. Application of adaptive neuro-fuzzy inference system for vigilance level estimation by using wavelet-entropy feature extraction. Expert Systems with Applications. 2009;36(4):7390-9.

30.Akin M, Kurt MB, Sezgin N, Bayram M. Estimating vigilance level by using EEG and EMG signals. Neural Computing and Applications. 2008;17(3):227-36.

31.Schmidt EA, Schrauf M, Simon M, Fritzsche M, Buchner A, Kincses WE. Drivers' misjudgement of vigilance state during prolonged monotonous daytime driving. Accident Analysis \& Prevention. 2009;41(5):1087-93.

32.Subasi A. Automatic recognition of alertness level from EEG by using neural network and wavelet coefficients. Expert systems with applications. 2005;28(4):701-11.

33.Kaber DB, Endsley MR. Out-of-the-loop performance problems and the use of intermediate levels of automation for improved control system functioning and safety. Process Safety Progress. 1997;16(3):126-31.

34.Kaber DB, Perry CM, Segall N, McClernon CK, Prinzel III LJ. Situation awareness implications of adaptive
2017;25:1796-805.

6.Gagnon J-F, Gagnon O, Lafond D, Parent M, Tremblay S, editors. A Systematic Assessment of Operational Metrics for Modeling Operator Functional State. PhyCS; 2016.

7.DiDomenico A, Nussbaum MA. Interactive effects of physical and mental workload on subjective workload assessment. International journal of industrial ergonomics. 2008;38(11-12):977-83.

8.Teh E, Jamson S, Carsten O, Jamson H. Temporal fluctuations in driving demand: The effect of traffic complexity on subjective measures of workload and driving performance. Transportation research part F: traffic psychology and behaviour. 2014;22:207-17.

9.DiDomenico A, Nussbaum MA. Effects of different physical workload parameters on mental workload and performance. International Journal of Industrial Ergonomics. 2011;41(3):255-60.

10.Stanton NA, Hedge A, Brookhuis K, Salas E, Hendrick HW. Handbook of human factors and ergonomics methods: CRC press; 2004.

11.Sterman M, Mann C. Concepts and applications of EEG analysis in aviation performance evaluation. Biological psychology. 1995;40(1-2):115-30.

12.Ryu K, Myung R. Evaluation of mental workload with a combined measure based on physiological indices during a dual task of tracking and mental arithmetic. International Journal of Industrial Ergonomics. 2005;35(11):991-1009.

13. Patka S, Khatib M.S, Kelwade K. Recent Trends and Rapid Development of Applications In Data Mining. IOSR Journal of Computer Science. 2014:73-8.

14.Papantoniou P. Risk factors, driver behaviour and accident probability. The case of distracted driving: National Technical University of Athens; 2015.

15.Scerbo MW, Parasuraman R, Mouloua M. Theoretical perspectives on adaptive automation. 1996.

16.Byrne EA, Parasuraman R. Psychophysiology and adaptive automation. Biological psychology. 1996;42(3):24968.

17.Wickens CD, Hollands JG, Banbury S, Parasuraman R. Engineering psychology and human performance: Psychology Press; 2015.

18.Cannon JA, Krokhmal PA, Lenth RV, Murphey R. An algorithm for online detection of temporal changes in operator cognitive state using real-time psychophysiological data. Biomedical Signal Processing and Control. 2010;5(3):229-36.

19.Cannon J, Krokhmal PA, Chen Y, Murphey R. Detection of temporal changes in psychophysiological data using statistical process control methods. Computer methods and programs in biomedicine. 2012;107(3):367-81.

20.Kramer AF. Physiological metrics of mental workload: A 
47.Azim T, Jaffar MA, Mirza AM, editors. Automatic fatigue detection of drivers through pupil detection and yawning analysis. 2009 Fourth International Conference on Innovative Computing, Information and Control (ICICIC); 2009: IEEE.(Persian).

48. Qin H, Liu J, Hong T, editors. An eye state identification method based on the Embedded Hidden Markov Model. 2012 IEEE International Conference on Vehicular Electronics and Safety (ICVES 2012); 2012: IEEE.

49.Lan P, Ji Q, Looney CG, editors. Information fusion with Bayesian networks for monitoring human fatigue. Proceedings of the Fifth International Conference on Information Fusion FUSION 2002(IEEE Cat No 02EX5997); 2002: IEEE.

50.Ji Q, Zhu Z, Lan P. Real-time nonintrusive monitoring and prediction of driver fatigue. IEEE transactions on vehicular technology. 2004;53(4):1052-68.

51.King L, Nguyen HT, Lal S, editors. Early driver fatigue detection from electroencephalography signals using artificial neural networks. 2006 International Conference of the IEEE Engineering in Medicine and Biology Society; 2006: IEEE.

52.Bundele MM, Banerjee R, editors. Detection of fatigue of vehicular driver using skin conductance and oximetry pulse: a neural network approach. Proceedings of the 11th International Conference on Information Integration and web-based applications \& services; 2009: ACM.

53.Yeo MV, Li X, Shen K, Wilder-Smith EP. Can SVM be used for automatic EEG detection of drowsiness during car driving? Safety Science. 2009;47(1):115-24.

54.Li G, Chung W-Y. Detection of driver drowsiness using wavelet analysis of heart rate variability and a support vector machine classifier. Sensors. 2013;13(12):16494511.

55.Lal SK, Craig A. Driver fatigue: electroencephalography and psychological assessment.Psychophysiology.2002;39(3):313-21.

56.Takahashi I, Yokoyama K, editors. Development of a feedback stimulation for drowsy driver using heartbeat rhythms. 2011 Annual International Conference of the IEEE Engineering in Medicine and Biology Society; 2011: IEEE.

57.Zhu Z, Ji Q, editors. Real time and non-intrusive driver fatigue monitoring. Proceedings The 7 th International IEEE Conference on Intelligent Transportation Systems (IEEE Cat No 04TH8749); 2004: IEEE.

58.Patel M, Lal SK, Kavanagh D, Rossiter P. Applying neural network analysis on heart rate variability data to assess driver fatigue. Expert systems with Applications. 2011;38(6):7235-42.

59.Khushaba RN, Kodagoda S, Lal S, Dissanayake G. Driver drowsiness classification using fuzzy wavelet-packet- automation for information processing in an air traffic control-related task. International Journal of Industrial Ergonomics. 2006;36(5):447-62.

35.Zhang J-H, Wang X-Y, Mahfouf M, Linkens DA, editors. Fuzzy logic based identification of operator functional states using multiple physiological and performance measures. 2008 International Conference on BioMedical Engineering and Informatics; 2008: IEEE.

36.Zhang J-H, Xia J-J, Garibaldi JM, Groumpos PP, Wang R-B. Modeling and control of operator functional state in a unified framework of fuzzy inference petri nets. Computer methods and programs in biomedicine. 2017;144:147-63.

37.Hwang S-L, Yau Y-J, Lin Y-T, Chen J-H, Huang T-H, Yenn T-C, et al. Predicting work performance in nuclear power plants. Safety science. 2008;46(7):1115-24.

38. Hachicha W, Ghorbel A. A survey of control-chart pattern-recognition literature (1991-2010) based on a new conceptual classification scheme. Computers \& Industrial Engineering. 2012;63(1):204-22.

39.Saffari M, Sanaeinasab H, Pakpour AH. How to Do a Systematic Review Regard to Health: A Narrative Review. Iranian Journal of Health Education and Health Promotion. 2013;1(1):51-61.(Persian).

40.Ahmadi M, Sedighie L, Rohani C. Evidence-based review in the field of developing critical appraisal tools for quantitative studies. Scientific Journal of Nursing, Midwifery and Paramedical Faculty. 2018;3(3):57-72. (Persian).

41.Rohani C, Gholamnejad H. Critical appraisal on the reporting of Nested Case-Control Studies according to the STROBE statement. Iranian Journal of Nursing Research. 2016;10(4):20-31.

42.Yin B-C, Fan X, Sun Y-F. Multiscale dynamic features based driver fatigue detection. International Journal of Pattern Recognition and Artificial Intelligence. 2009;23(03):575-89.

43.Xie J-F, Xie M, Zhu W, editors. Driver fatigue detection based on head gesture and PERCLOS. 2012 International Conference on Wavelet Active Media Technology and Information Processing (ICWAMTIP) ; 2012: IEEE.

44.Tadesse E, Sheng W, Liu M, editors. Driver drowsiness detection through HMM based dynamic modeling. 2014 IEEE International conference on robotics and automation (ICRA); 2014: IEEE.

45.Coetzer RC, Hancke GP, editors. Eye detection for a real-time vehicle driver fatigue monitoring system. 2011 IEEE Intelligent Vehicles Symposium (IV); 2011: IEEE.

46.Saradadevi M, Bajaj P. Driver fatigue detection using mouth and yawning analysis. International journal of Computer science and network security. 2008;8(6):1838 . 
مرور سيستماتيك كاربرد روش هاى داده كاوى...

Systems with Applications. 2009;36(4):7651-8.

73.Brandt T, Stemmer R, Rakotonirainy A, editors. Affordable visual driver monitoring system for fatigue and monotony. 2004 IEEE International Conference on Systems, Man and Cybernetics (IEEE Cat No 04CH37583); 2004: IEEE.

74.Jin S, Park S-Y, Lee J-J. Driver fatigue detection using a genetic algorithm. Artificial Life and Robotics. 2007;11(1):87-90.

75.Mbouna RO, Kong SG, Chun M-G. Visual analysis of eye state and head pose for driver alertness monitoring. IEEE transactions on intelligent transportation systems. 2013;14(3):1462-9.

76.Lee B-G, Chung W-Y. Driver alertness monitoring using fusion of facial features and bio-signals. IEEE Sensors Journal. 2012;12(7):2416-22.

77.Yang G, Lin Y, Bhattacharya P. A driver fatigue recognition model based on information fusion and dynamic Bayesian network. Information Sciences. 2010;180(10):1942-54.

78.Azman A, Meng Q, Edirisinghe E, editors. Non intrusive physiological measurement for driver cognitive distraction detection: Eye and mouth movements. 2010 3rd International Conference on Advanced Computer Theory and Engineering (ICACTE); 2010: IEEE.

79.Kutila M, Jokela M, Markkula G, Rué MR, editors. Driver distraction detection with a camera vision system. 2007 IEEE International Conference on Image Processing; 2007: IEEE.

80.Zhang H, Zhu Y, Maniyeri J, Guan C, editors. Detection of variations in cognitive workload using multi-modality physiological sensors and a large margin unbiased regression machine. 2014 36th Annual International Conference of the IEEE Engineering in Medicine and Biology Society; 2014: IEEE.

81.Miyaji M, Kawanaka H, Oguri K, editors. Effect of pattern recognition features on detection for driver's cognitive distraction. 13th International IEEE Conference on Intelligent Transportation Systems; 2010: IEEE.

82.Miyaji M, Danno M, Kawanaka H, Oguri K, editors. Driver's cognitive distraction detection using AdaBoost on pattern recognition basis. 2008 IEEE International Conference on Vehicular Electronics and Safety; 2008: IEEE.

83.Tango F, Botta M, editors. Evaluation of distraction in a driver-vehicle-environment framework: An application of different data-mining techniques. Industrial Conference on Data Mining; 2009: Springer.

84.Zhang Y, Owechko Y, Zhang J, editors. Driver cognitive workload estimation: A data-driven perspective. Proceedings The 7th International IEEE Conference on Intelligent Transportation Systems (IEEE Cat No based feature-extraction algorithm. IEEE Transactions on Biomedical Engineering. 2010;58(1):121-31.

60.Kim Y, Kim Y, Hahn M, editors. Detecting driver fatigue based on the driver's response pattern and the front view environment of an automobile. 2008 Second International Symposium on Universal Communication; 2008: IEEE.

61.Vural E, Cetin M, Ercil A, Littlewort G, Bartlett M, Movellan J, editors. Drowsy driver detection through facial movement analysis. International Workshop on Human-Computer Interaction: Springer. 2007:6-18.

62.Vural E, Çetin M, Erçil A, Littlewort G, Bartlett M, Movellan J. Machine learning systems for detecting driver drowsiness. In-vehicle corpus and signal processing for driver behavior: Springer:2009:97-110.

63.Rong-Ben W, Ke-You G, Shu-Ming S, Jiang-Wei C, editors. A monitoring method of driver fatigue behavior based on machine vision. IEEE IV2003 Intelligent Vehicles Symposium Proceedings (Cat No 03TH8683); 2003: IEEE.

64.Fan X, Yin B, Sun Y, editors. Nonintrusive driver fatigue detection. 2008 IEEE International Conference on Networking, Sensing and Control; 2008: IEEE.

65.Wang P, Shen L, editors. A method of detecting driver drowsiness state based on multi-features of face. 2012 5th International Congress on Image and Signal Processing; 2012: IEEE.

66.Luo X-q, Hu R, Fan T-e, editors. The driver fatigue monitoring system based on face recognition technology. 2013 Fourth International Conference on Intelligent Control and Information Processing (ICICIP); 2013: IEEE.

67.Sun C, Li JH, Song Y, Jin L, editors. Real-time driver fatigue detection based on eye state recognition. Applied Mechanics and Materials; 2014: Trans Tech Publ.

68. Tayade MR, Jeyakumar A, Kore AB, Galshetwar VM. Real Time Eye State Monitoring System for Driver Drowsiness Detection. International Journal of Emerging Technology and Advanced Engineering. 2014;4(6):452-6.

69.Senaratne R, Hardy D, Vanderaa B, Halgamuge S, editors. Driver fatigue detection by fusing multiple cues. International Symposium on Neural Networks; 2007: Springer.

70.Wu J-D, Chen T-R. Development of a drowsiness warning system based on the fuzzy logic images analysis. Expert Systems with Applications. 2008;34(2):1556-61.

71.Bagci A, Ansari R, Khokhar A, Cetin E, editors. Eye tracking using Markov models. Proceedings of the 17th International Conference on Pattern Recognition, 2004 ICPR 2004; 2004: IEEE.

72.Hu S, Zheng G. Driver drowsiness detection with eyelid related parameters by Support Vector Machine. Expert 
\& Prevention. 1999;31(3):199-211.

97.Dubart D, Loslever P, Popieul J-C, Moessinger M. Multiple Correspondence Analysis of Fuzzyfied Task Performance and Psycho-Physiological Test Data: Use in Real Car Following Situations. IFAC Proceedings Volumes. 2013;46(15):455-60.

98.Wilson GF, Russell CA. Operator functional state classification using multiple psychophysiological features in an air traffic control task. Human Factors. 2003;45(3):3819.

99.Hannula M, Huttunen K, Koskelo J, Laitinen T, Leino T. Comparison between artificial neural network and multilinear regression models in an evaluation of cognitive workload in a flight simulator. Computers in biology and medicine. 2008;38(11-12):1163-70.

100.Torres-Salomao LA, Mahfouf M, Obajemu O. Interval type-2 fuzzy logic adaptive modelling for human operators undergoing mental stress. IFAC Proceedings Volumes. 2014;47(3):9880-5.

101.Gianazza D. Forecasting workload and airspace configuration with neural networks and tree search methods. Artificial intelligence. 2010;174(7-8):530-49.

102.Gregoriades A, Sutcliffe A. Workload prediction for improved design and reliability of complex systems. Reliability Engineering \& System Safety. 2008;93(4):530-49.

103.Zhang G, Wang W, Pepe A, Xu R, Schnell T, Anderson $\mathrm{N}$, et al. A systematic approach for real-time operator functional state assessment. 2012:190-199.

104.Wilson GF, Russell CA. Real-time assessment of mental workload using psychophysiological measures and artificial neural networks. Human factors. 2003;45(4):63544.

105.Yin Z, Zhang J. Operator functional state classification using least-square support vector machine based recursive feature elimination technique. Computer methods and programs in biomedicine. 2014;113(1):101-15.

106.Yin Z, Zhang J. Cross-session classification of mental workload levels using EEG and an adaptive deep learning model. Biomedical Signal Processing and Control. 2017;33:30-47.

107.Wang R, Zhang J, Zhang Y, Wang X. Assessment of human operator functional state using a novel differential evolution optimization based adaptive fuzzy model. Biomedical Signal Processing and Control. 2012;7(5):490-8. 108.Zhang J, Mahfouf M, Linkens D, Nickel P, Hockey G, editors. Adaptive fuzzy model of operator functional state in human-machine system: a preliminary study. Proceedings of the IASTED international conference; 2007. 109.Xia J, Zhang J, Wang R. Regulation of Adaptive HumanMachine System Based on Fuzzy Inference Petri Net. IFAC-PapersOnLine. 2015;48(20):475-80.

110.Ting C-H, Mahfouf M, Nassef A, Linkens DA, Panout-
04TH8749); 2004: IEEE.

85.Craven PL, Belov N, Tremoulet P, Thomas M, Berka C, Levendowski D, et al. Cognitive workload gauge development: comparison of real-time classification methods. Foundations of Augmented Cognition. 2006:75-84.

86.Miyaji M, Kawanaka H, Oguri K, editors. Driver's cognitive distraction detection using physiological features by the adaboost. 2009 12th International IEEE conference on intelligent transportation systems; 2009: IEEE.

87.Solovey ET, Zec M, Garcia Perez EA, Reimer B, Mehler B, editors. Classifying driver workload using physiological and driving performance data: two field studies. Proceedings of the SIGCHI Conference on Human Factors in Computing Systems; 2014: ACM.

88.Tjolleng A, Jung K, Hong W, Lee W, Lee B, You H, et al. Classification of a Driver's cognitive workload levels using artificial neural network on ECG signals. Applied ergonomics. 2017;59:326-32.

89.Liao W, Zhang W, Zhu Z, Ji Q, editors. A real-time human stress monitoring system using dynamic Bayesian network. 2005 IEEE Computer Society Conference on Computer Vision and Pattern Recognition (CVPR'05)Workshops; 2005: IEEE.

90.Wijnands JS, Thompson J, Aschwanden GD, Stevenson $M$. Identifying behavioural change among drivers using Long Short-Term Memory recurrent neural networks. Transportation research part F: traffic psychology and behaviour. 2018;53:34-49.

91.Gu H, Ji Q, editors. Facial event classification with task oriented dynamic Bayesian network. Proceedings of the 2004 IEEE Computer Society Conference on Computer Vision and Pattern Recognition, 2004 CVPR 2004; 2004 : IEEE.

92.Sabet M, Zoroofi RA, Sadeghniiat-Haghighi K, Sabbaghian $\mathrm{M}$, editors. A new system for driver drowsiness and distraction detection. 20th Iranian Conference on Electrical Engineering (ICEE2012); 2012: IEEE.(Persian).

93.Sigari MH, editor Driver hypo-vigilance detection based on eyelid behavior. 2009 seventh international conference on advances in pattern recognition; 2009: IEEE. (Persian).

94.Muñoz M, Reimer B, Lee J, Mehler B, Fridman L. Distinguishing patterns in drivers' visual attention allocation using Hidden Markov Models. Transportation research part F: traffic psychology and behaviour. 2016;43:90103.

95.Rumschlag G, Palumbo T, Martin A, Head D, George R, Commissaris RL. The effects of texting on driving performance in a driving simulator: The influence of driver age. Accident Analysis \& Prevention. 2015;74:145-9.

96.Verwey WB, Zaidel DM. Preventing drowsiness accidents by an alertness maintenance device. Accident Analysis 
مرور سيستماتيك كاربرد روش هاى داده كاوى...

2014;8:703.

119.Noel JB, Bauer Jr KW, Lanning JW. Improving pilot mental workload classification through feature exploitation and combination: a feasibility study. Computers \& operations research. 2005;32(10):2713-30.

120. Thakur GS, Gupta A, Gupta S. Data mining for prediction of human performance capability in the softwareindustry. International Journal of Data-Mining and Knowledge Management Process (IJDKP). 2015.

121.Currie DJ, Smith C, Jagals P. The application of system dynamics modelling to environmental health decisionmaking and policy-a scoping review. BMC public health. 2018;18(1):402.

122. Mohammadi Z. Dynamic modeling of mental performance using artificial neural network and considering the psychoacoustic aspects. PhD thesis: Shahid Beheshti university of medical sciences; 2020.(Persian).

123.Arekhi S, Adibnejad M. Efficiency assessment of the of Support Vector Machines for land use classification using Landsat ETM+ data (Case study: Ilam Dam Catchment). Iranian Journal of Range and Desert Research. 2011;18(3):420-40.(Persian).

124.Hegde J, Rokseth B. Applications of machine learning methods for engineering risk assessment-A review. Safety science. 2020;122:104492.

125.Sedehi M, Mehrabi Y, Kazemnejad A, Hadaegh F. Comparison of artificial neural network, logistic regression and discriminant analysis methods in prediction of metabolic syndrome. Iranian journal of endocrinology and metabolism. 2009;11(6):638-646. (Persian).

126.Sepehri M, Iildoromi AR, Hosseini SZ, Nori H, Mohammadzadeh F, Artimani MM. Combination of Neural Networks and Genetic Algorithms, an Approach to Estimate the Flood Flow. Iranian journal of watershed management science and engineering. 2018;11(39):2328. (Persian).

127. Kheirabadi GR, Bolhari J. Role of human factors in road accidents. 2012. (Persian). sos G, Nickel P, et al. Real-time adaptive automation system based on identification of operator functional state in simulated process control operations. IEEE Transactions on Systems, Man, and Cybernetics-Part A: Systems and Humans. 2009;40(2):251-62.

111.Yin Z, Zhang J, Wang R. Neurophysiological featurebased detection of mental workload by ensemble support vector machines. Advances in Cognitive Neurodynamics (V): Springer; 2016:469-75.

112.Christensen JC, Estepp JR, Wilson GF, Russell CA. The effects of day-to-day variability of physiological data on operator functional state classification. NeuroImage. 2012;59(1):57-63.

113.Zhang C, Zheng C-X, Yu X-L. Automatic recognition of cognitive fatigue from physiological indices by using wavelet packet transform and kernel learning algorithms. Expert Systems with Applications. 2009;36(3):4664-71.

114.Chen L-1, Zhao Y, Zhang J, Zou J-z. Automatic detection of alertness/drowsiness from physiological signals using wavelet-based nonlinear features and machine learning. Expert Systems with Applications. 2015;42(21):7344-55.

115.Laurent F, Valderrama M, Besserve M, Guillard M, Lachaux J-P, Martinerie J, et al. Multimodal information improves the rapid detection of mental fatigue. Biomedical Signal Processing and Control. 2013;8(4):400-8.

116. Moon B, Lee H, Lee Y, Park J, Oh I, Lee J. Fuzzy systems to process ECG and EEG signals for quantification of the mental workload. Information Sciences. 2002;142(14):23-35.

117. Nikolova R, Radev V, Vukov M. Functional determination of the operator state in the interaction of humans with automated systems. National center of hygiene Sofia (Bulgaria) medical ecology and nutrition, 2003.

118.Ke Y, Qi H, He F, Liu S, Zhao X, Zhou P, et al. An EEG-based mental workload estimator trained on working memory task can work well under simulated multi-attribute task. Frontiers in human neuroscience. 